\title{
Nonagons in the Hagia Sophia and the Selimiye Mosque
}

\author{
Antonia Redondo Buitrago • Dirk Huylebrouck
}

Published online: 28 January 2015

(C) Kim Williams Books, Turin 2015

\begin{abstract}
One of the classical topics in geometry is the ruler and compass construction of good approximations of the regular nonagon. We propose a method to choose a desired error of approximation, based on new linear third-order recurrence relations. It is related to patterns shown on the balustrade of minbar of Selimiye Mosque in Edirne (Turkey, 1569-1575), where apparently regular nonagons are placed over a net of regular hexagons. This kind of ornament also occurs in Hagia Sophia in the minbar, in freezes stucco carvings and in window grilles. In Medieval Islamic art and architecture the use of the nonagon is not frequent, but it is remarkable that this particular grid of interlocked nonagons and hexagons appears in the decorations of the works of Ottoman architect Mimar Sinan. The pattern is present in his masterpiece, the Selimiye Mosque, in the Hagia Sophia and in other works in the Istanbul city. Looking at practical ways for the construction of the pattern, we provide simple procedures to obtain angles close to $40^{\circ}$ that could have been useful for a craftsman to realize the nonagonal geometric designs. In particular, almost regular nonagons are constructed using some elementary shapes that are related with semi-regular tessellations. We compare the patterns obtained through theoretical considerations to those displayed in the examples given above. Several hypotheses are proposed for the practical construction of the interlocked hexagonal patterns for nonagons.
\end{abstract}

Keywords Ruler and compass construction · Nonagon - Selimiye Mosque $\cdot$ Hagia Sophia $\cdot$ Minbar · Mimar Sinan

\footnotetext{
A. Redondo Buitrago

Departamento de Matemáticas, I.E.S. Bachiller Sabuco, Avenida de España, 9, 02002 Albacete, Spain

e-mail: aredondo@sabuco.com

D. Huylebrouck $(\bowtie)$

LUCA School of Arts KU Leuven, Paleizenstraat 65, 1030 Brussels, Belgium

e-mail: Huylebrouck@gmail.com
}

Birkhäuser 


\section{Introduction}

A classical Greek topic in geometry was to determine the regular polygons that are constructible by compass and ruler, in a finite number of steps. The equilateral triangle, the square, the pentagon and the hexagon all are constructible, but heptagon is not, nor is the nonagon. A related classical topic is the ruler and compass construction of the angle of $40^{\circ}$, that is, the angle in the centre of a nonagon pointing to two adjacent vertices. In the late eleventh century, Muslim architects and mathematicians discovered some approximate constructions of the regular nonagon. Indeed, in the Seljuk period (1038-1194) and in Mamluk architecture (1250-1517) geometric decorations show mainly patterns with stars with five, six, eight and ten points, as well as twelve and sixteen, although some stars with seven and nine points appear as well, and even some stars with eleven and thirteen points (Fig. 1).

Later on, some nine-pointed stars appear (in other words, concave eighteen-sided polygons) made by connecting vertices of other constructible polygons. However, the regular convex nonagon is not frequently determined by these methods. Almost regular nonagons placed over a net of regular hexagons can be found in the balustrade leading to the minbar of the Selimiye Mosque in Edirne (Turkey, 1569-1575; Fig. 2).

This kind of ornament also occurs in Hagia Sophia in the top of the frieze and in the stucco carvings of the gallery or mahfil of Sultan Murad III (1574-1595; Fig. 3), in the grill on the upper level of the Library of Sultan Mahmud I (1739; Fig. 4), and in the minbar in the rosette decorating the end of the staircase (Fig. 5).

The use of the nonagon is not frequent. It is remarkable that this particular grid of interlocking nonagons and hexagons often appears in the decorations of the works of Ottoman architect Mimar Sinan. Decorations similar to those in the Selimiye Mosque and the Hagia Sophia can be seen in his mausoleum of Shehzade Mehmet

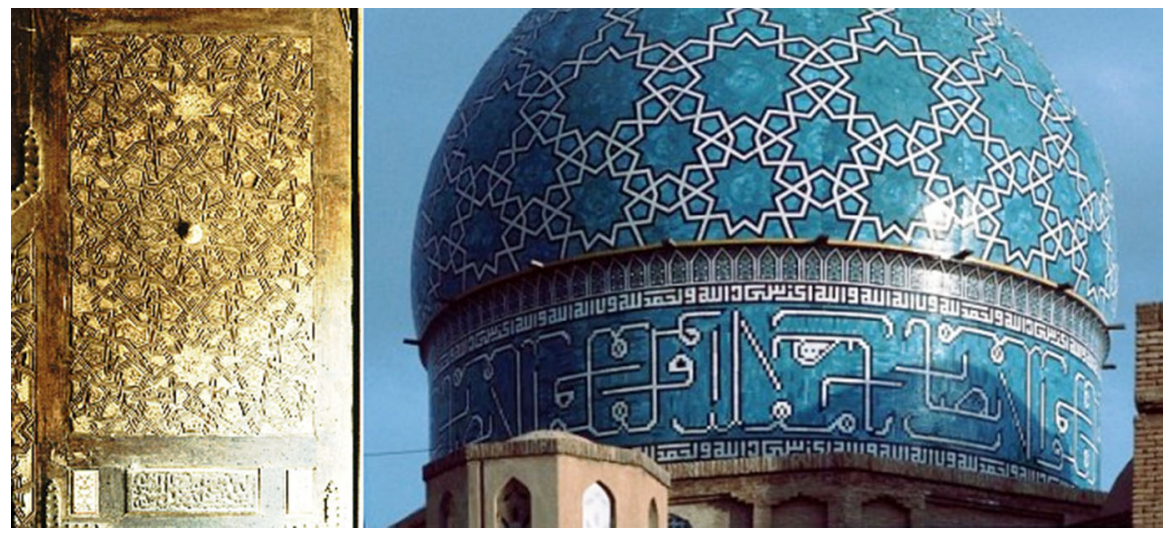

Fig. 1 a Left Mamluk woodwork on the minbar in al-Ghuriyya Complex, Cairo, Egypt (1505) (Wade Photo Archive EGY1722); b right star dome of the Shrine of Shah Ni'Matuollahi in Mahan, Iran (1436) (WADE Photo Archive IRA 2313) 

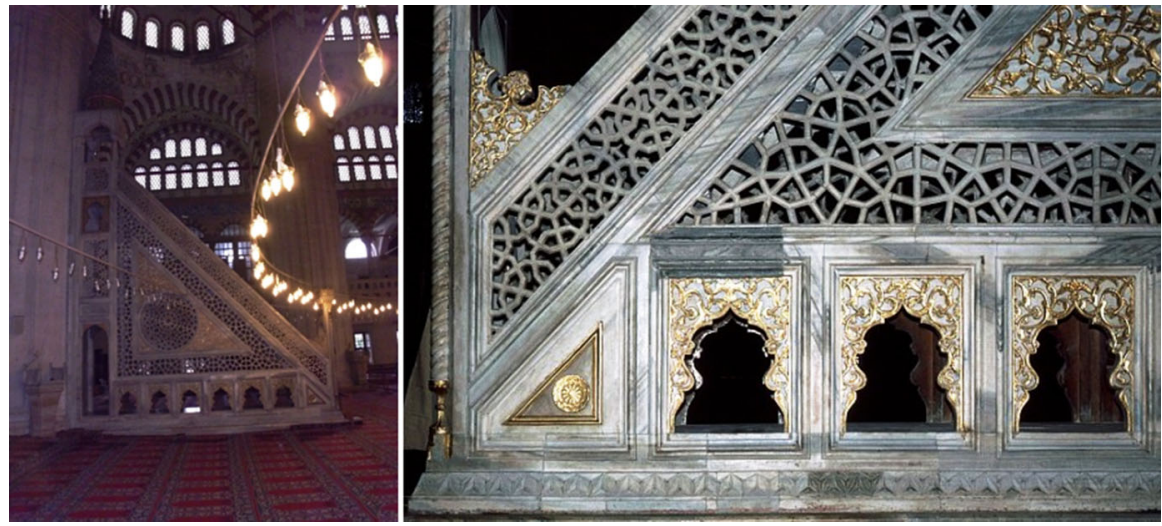

Fig. 2 Minbar of the Selimiye Mosque in Edirne (Wikimedia Commons) and detail of the decoration in woodwork carved (WADE Photo Archive TUR 0117)

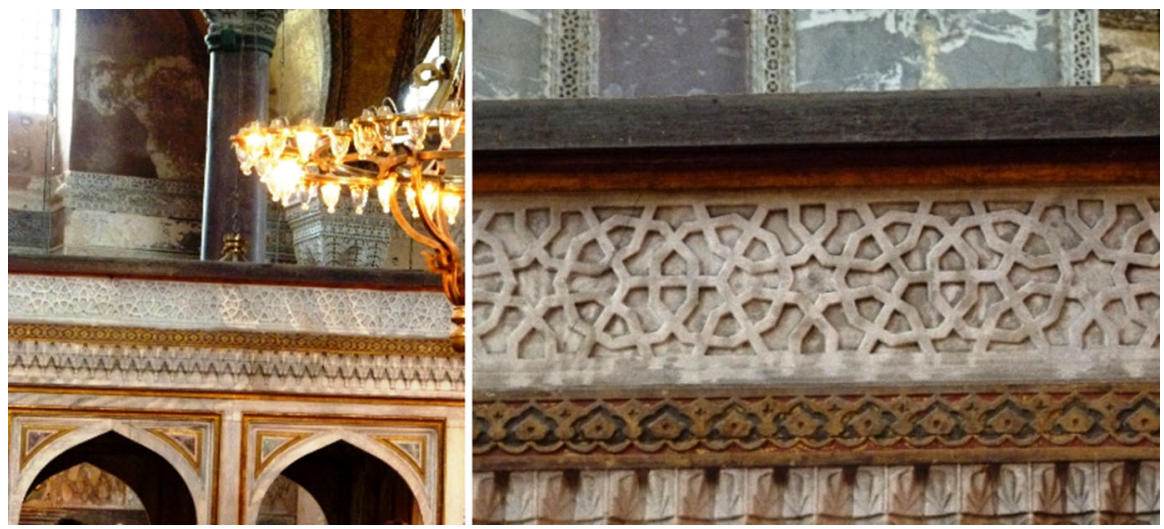

Fig. 3 a Left Muezzin galleryor mahfil, Hagia Sophia; b right a detail of the stucco carving in the frieze. Photos: authors

in Istanbul (1557; Fig. 6) and in the balcony of the minaret in the Mihrimah Sultan Camii in Uskudar, Istanbul (1546-1548). Also in Istanbul, the pattern occurs in the mausoleum of Koca Sinan Paşa (1596) by Mimar Davud A ğa, a student of Mimar Sinan. Surprisingly, the pattern appears in India too, in a panel decorating the outside of the Red Fort in Agra, built by the Mughal dynasty in the time when the Ottoman Empire reached until India (1550 EC), and in the Jaypur Palace built during the Rajput dynasty (Fig. 7).

This paper studies the geometry of this nonagonal pattern. It presents original results for the regular nonagon and looks at practical ways to approximate its construction. It gives simple procedures for obtaining angles close to $40^{\circ}$ that could have been useful for craftsmen to realize the nonagonal geometric patterns. Observation of the patterns provides mathematical arguments to analyse the 


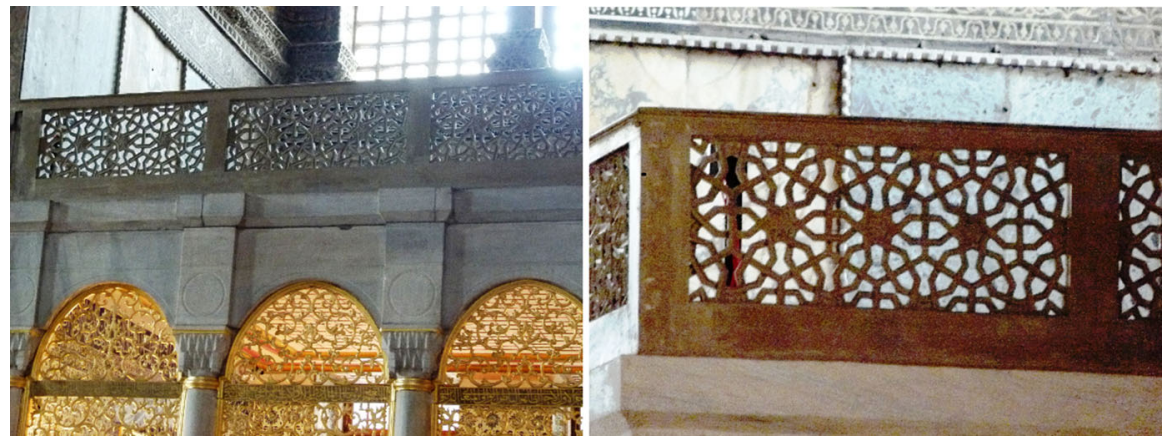

Fig. 4 a Left the Library of Sultan Mahmum I, Hagia Sophia; b right detail of the grill on the upper level. Photos: authors
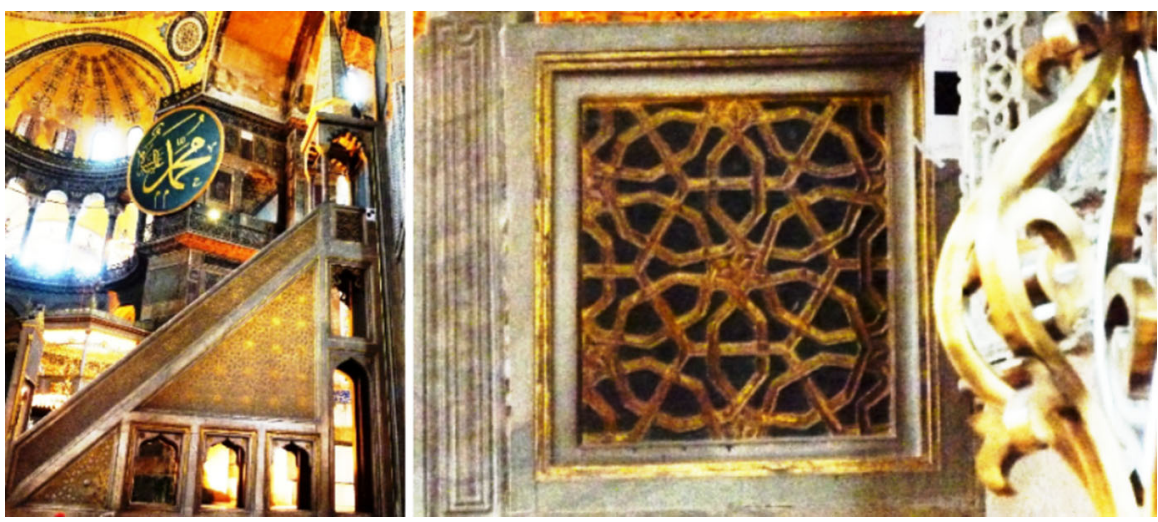

Fig. 5 a Left Minbar of Hagia Sophia; b right detail of the rosette in the panel decorating the end of the stairway. Photos: authors

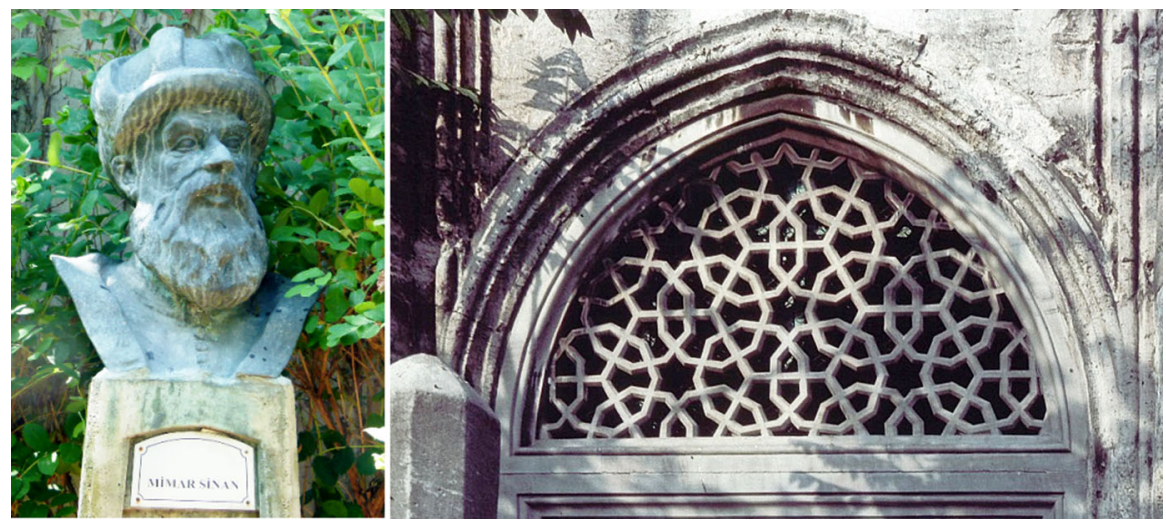

Fig. 6 a Left Sculpture of Master Sinan in the garden of the MET University, Ankara. Photo: authors; b right window grill of the Mausoleum of Shehzade Mehmet, Istanbul (WADE Photo Archive TUR 1130) 

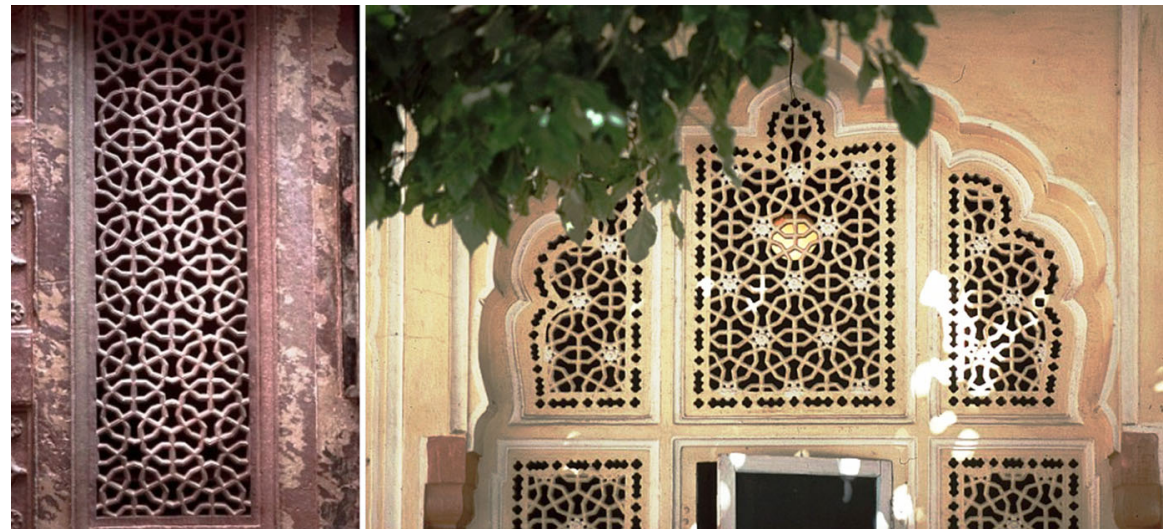

Fig. 7 a Left Red Fort, Agra, India, 1550 EC (WADE Photo Archive IND 0336); b right Maharajah's Palace, Jaipur, India, 1700 EC (WADE Photo Archive IND1006)

hypothetical regularity of the nonagons of the pattern. Finally, the pattern will be constructed in different ways.

\section{Mathematical Prerequisites}

Given a regular nonagon $A B C D E F G H I$, the numbers $\alpha, \beta$ and $\gamma(\alpha<\beta<\gamma)$ usually denote the ratios between the three diagonals of the polygon and its side (see Fig. 8a). Without loss of generality, we will always consider regular nonagons with side $A B=1, \quad$ so that $A C=\alpha=1.87938 \ldots, \quad A D=\beta=2.53208 \ldots \quad$ and $A E=\gamma=2.87938 \ldots$

The six diagonals with common vertex $A$ dissect the nonagon in seven triangles $A B C, A C D, \ldots, A H I$, where $\angle B A C=\angle C A D=\cdots=\angle H A I=20^{\circ}$, so that $\angle B A E=$ $60^{\circ}$ and $\angle A B G=60^{\circ}$. Thus, the triangles $A B J, K D E$ and $H L G$ shown in Fig. $8 \mathrm{~b}$ are
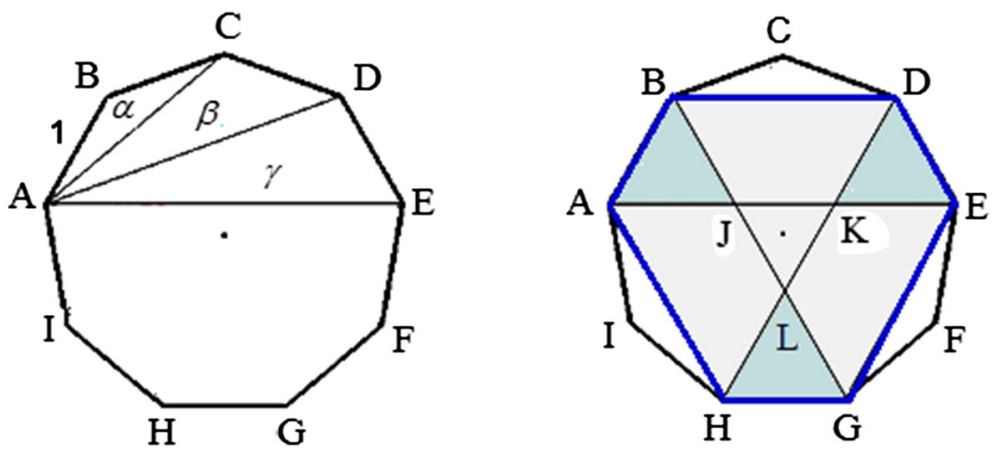

Fig. 8 a Left The three "diagonal/side" ratios of the regular nonagon; b right equiangular (though not necessarily regular) hexagon $A B D E G H$ and the derived equilateral nonagon 
equilateral with side 1 and the quadrilateral $A B D K$ is a parallelogram. Hence, Fig. $8 \mathrm{~b}$ is an illustration of the relation $\gamma=\alpha+1$.

In Fig. 8 we define an equiangular hexagon, $A B D E G H$, with angles $120^{\circ}$ and with sides of alternating lengths 1 and $\alpha$. Three congruent isosceles triangles, $C D B$, EFG and HIA, with sides 1,1 and $\alpha$ are added to it. Note that for any two lines segments of lengths $a$ and $b$, with $b / 2<a<b$, we can construct a general equiangular hexagon with shorter side $a$ and longer side $b$, deriving a nine-sided equilateral polygon whose regularity depends on the value of the ratio $R=b$ / $a$ where $1<R<2$ (Fig. 9). Thus, each value of $R$ generates an equilateral nonagon that will be regular only if $R=\alpha$. The case $a=b(R=1)$ is not considered because that equality implies the hexagon is regular, and then the derived nonagon is an equilateral triangle. The case $b / 2=a(R=2)$ is not considered because the nonagon derived in a similar way is the initial hexagon.

For each of these equilateral nonagons an equilateral triangle $P Q R$ is obtained by extending their sides $A B, D E$ and $H G$ (Fig. 10).

Since equilateral triangles allow covering the plane, equilateral convex nonagons and six-pointed stars may generate interesting tessellations using symmetries and equilateral triangles (Fig. 10). Elementary rosettes of these tessellations can be obtained by successive reflections with respect to one side, or equivalently, by successive rotations over $60^{\circ}$ with a vertex as centre.

An additional translation of the nonagons towards the centre of the rosette generates an interesting family of patterns (Fig. 11). In Fig. 12 we recognize the rosette of the grill and derived friezes shown in Figs. 2, 3, 4, 5 and on the right of Fig. 11 .

The nonagons of the patterns in the given examples are perceived as regular, but later we will examine how close this apparent regularity comes to an exact regularity. Equilateral nonagons with easily perceptible irregularity appear in the

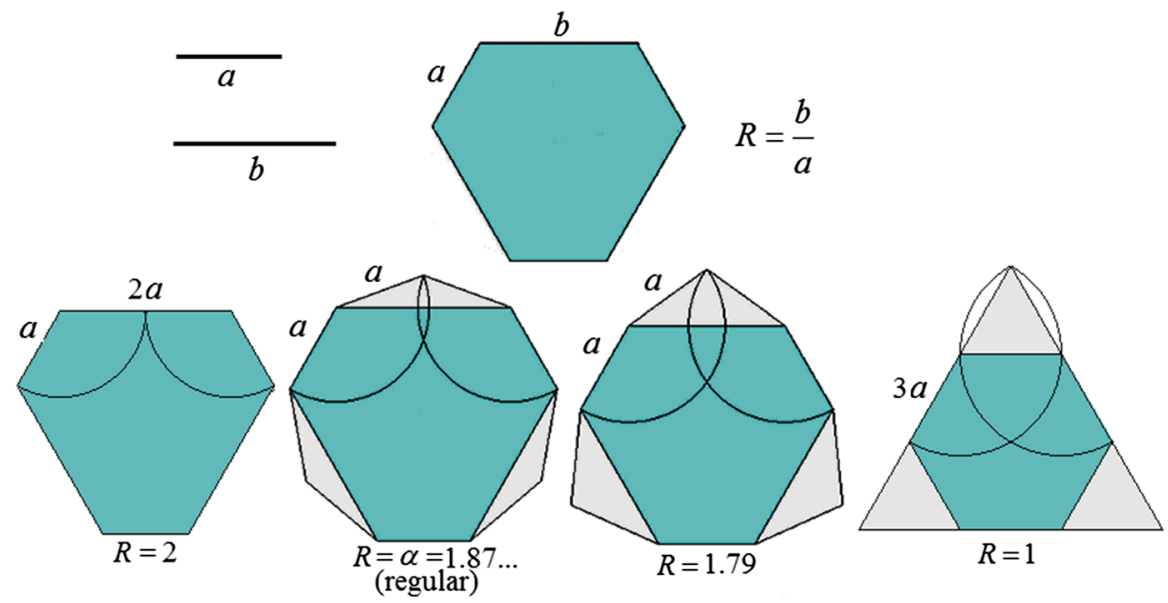

Fig. 9 The equiangular hexagon with alternating sides $a$ and $b$ (above) and equilateral nonagons for different values of $R$ (below) 

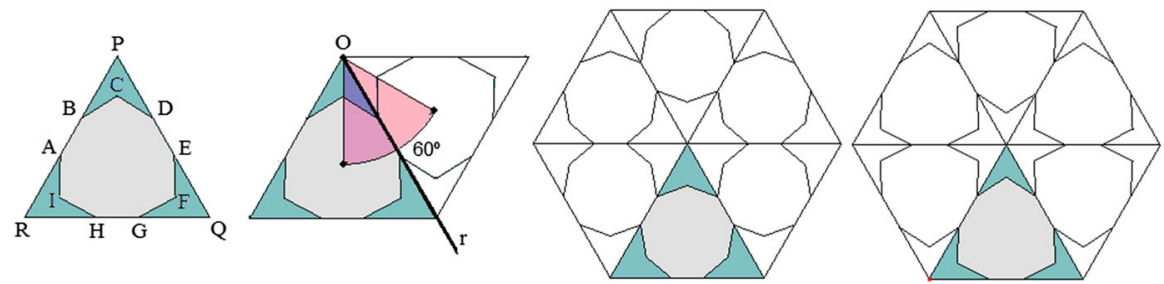

Fig. 10 Left equilateral triangle $P Q R$ generated by the nonagon and its common mirror image by reflection with respectto $\mathrm{r}$ or by a rotation over $60^{\circ}$ with centre the vertex $O$; right two rosettes
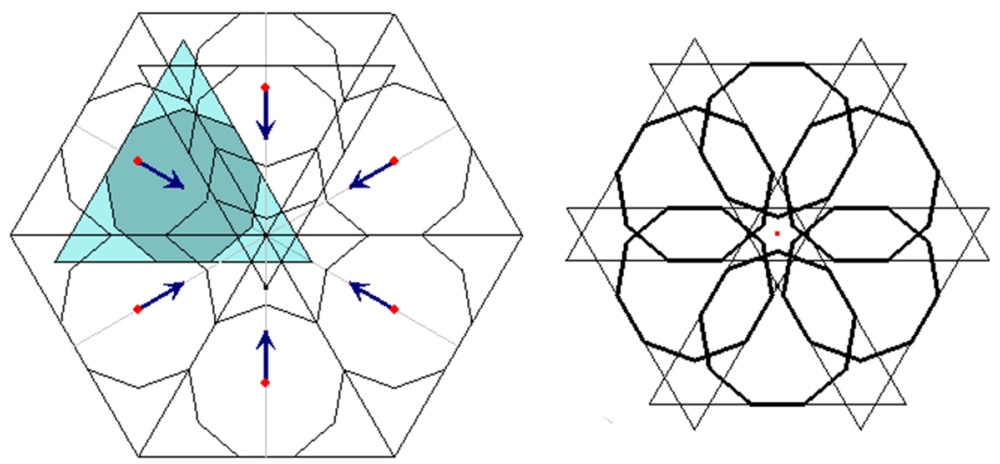

Fig. 11 Translation of the triangles yields a rosette with interlaced nonagons

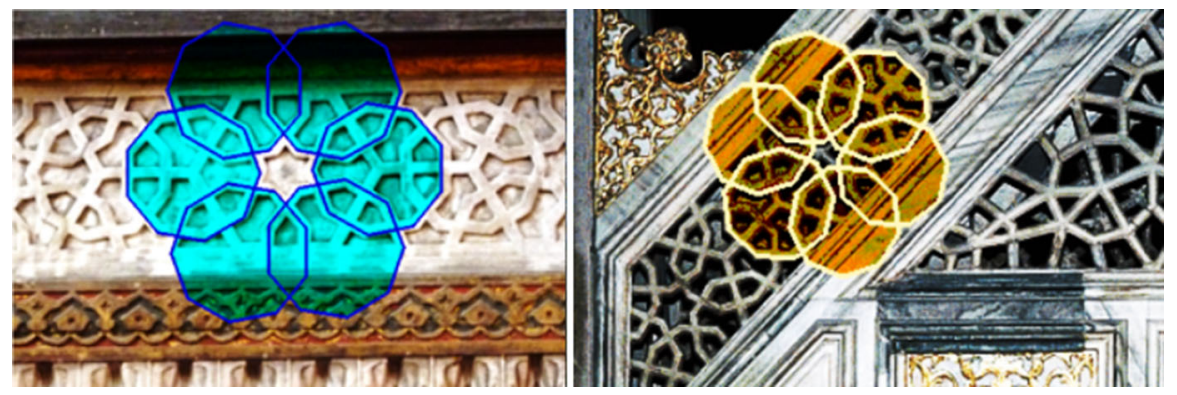

Fig. 12 Overlaying diagrams showing the rosette of interlocking nonagons obtained by the extension of polygons beyond the boundary of the frieze. Overlay sand photos: authors; see Figs. 2 and 3)

decoration in the tympanum of Gonbad-e Sork, Maragha, Iran, dated 1148 (Fig. 13). This unique intricate pattern shows a layer of interlaced non-regular nonagons shaped as on the right of Fig. 10, but with alternating up-down orientation. A layer of dodecagons is added, over a double hexagonal net. Although the variable orientation of the nonagons implies differences in the rosette as a whole, this decoration can be considered an example of the generation of nonagonal patterns by the given procedure. Figure 14 shows how the external ring of nonagons is the 

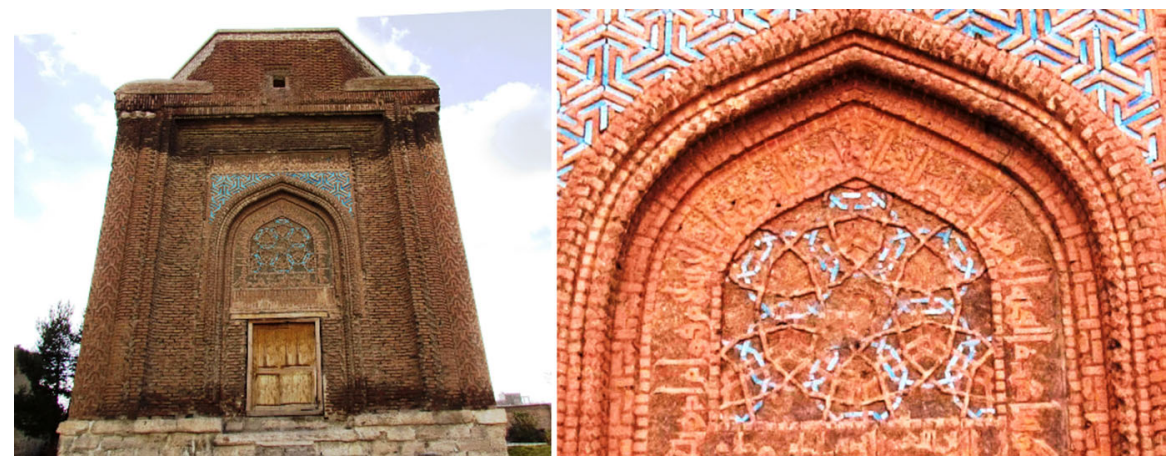

Fig. 13 a Left Gonbad-e Sork's tower in Maragha, Iran. Photo: Wikimedia Commons; b right detail
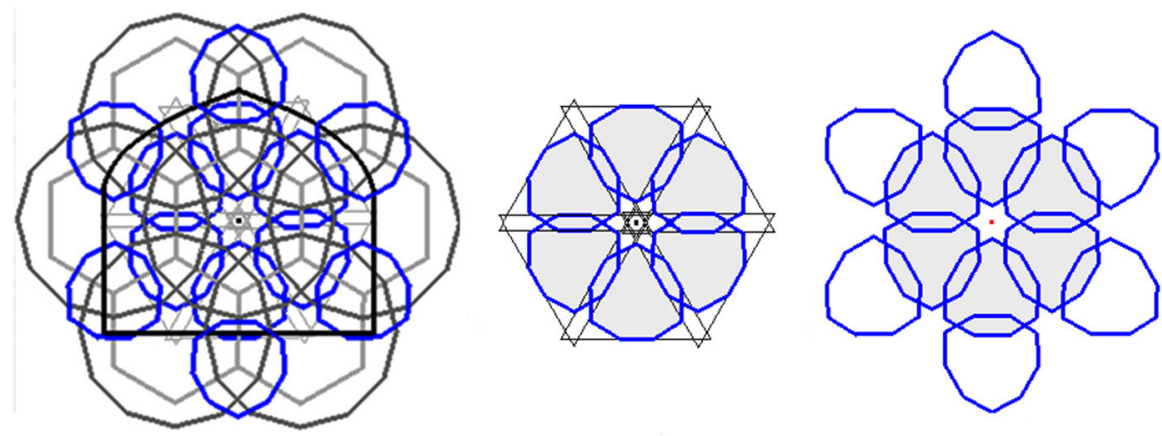

Fig. 14 Drawing by the authors of the decoration of the tympanus of Gonbad-e Sork's tower with the rosettes formed by irregular equilateral nonagons

reflection of the central rosette, which results after some translation of the nonagons of an initial rosette as given in the right of Fig. 10.

The ratios $\alpha, \beta$ and $\gamma$ satisfy a large number of relations between them (Steinbach 1997:26) thus generalizing the golden section. Sixteen different identities hold involving two or three of these ratios.

$$
\left.\begin{array}{c}
\frac{\alpha}{1}=\frac{1+\beta}{\alpha}=\frac{\alpha+\gamma}{\beta}=\frac{\beta+\gamma}{\gamma} \\
-\frac{\beta}{1}=\frac{\alpha+\gamma}{\alpha}=\frac{1+\beta+\gamma}{\beta}=\frac{\alpha+\beta+\gamma}{\gamma} \\
\frac{\gamma}{1}=\frac{\beta+\gamma}{\alpha}=\frac{\alpha+\beta+\gamma}{\beta}=\frac{1+\alpha+\beta+\gamma}{\gamma}
\end{array}\right\} \Rightarrow \begin{gathered}
\alpha^{2}-1-\beta=0 \\
\beta^{2}=1+\beta+\gamma \\
\gamma^{2}=1+\alpha+\beta+\gamma \\
\vdots
\end{gathered}
$$

None of these equalities is used in this work, since all of our results will be obtained from the identities $\gamma=\alpha+1, \alpha^{3}-3 \alpha-1=0$ and $\gamma^{3}-3 \gamma^{2}+1=0$. The relevance of equation $x^{3}-3 x-1=0$ in the construction of the regular nonagon was known to (Al-Biruni 1954). He showed that the chord of $40^{\circ}$ can be found if two cubic equations $x^{3}-3 x-1=0$ and $x^{3}-3 x+1=0$ can be solved (Hogendijk 1979). However, these equations are not independent and their solutions are related: the 
largest solution of the first equation is $\alpha=1.87938524 \ldots$, while the smallest solution of the second equation is $-\alpha$. So, both equations of Al-Biruni imply the identity $\alpha^{3}-3 \alpha-1=0$ :

$$
\begin{aligned}
x^{3}-3 x-1 & =0 \Rightarrow \alpha^{3}-3 \alpha-1=0 \Leftarrow-\alpha^{3}+3 \alpha+1=0 \\
& \Leftarrow(-\alpha)^{3}-3(-\alpha)+1=0 \Leftarrow x^{3}-3 x+1=0
\end{aligned}
$$

Also, because of the relation $\alpha=\gamma-1$, the substitution for $\alpha$ in the equality $\alpha^{3}-3 \alpha-1=0$, leads to the equality $\gamma^{3}-3 \gamma^{2}+1=0$.

Applying the cosine law to the triangle $A B C$ in Fig. 8 it follows that $\alpha=2 \cos 20^{\circ}$. Considering the triangles $A C D$ and $A D E$, this property, combined with the sine and cosine law, implies that $\beta^{2}=\alpha^{2}+1+\alpha$ and $\sin \left(20^{\circ}\right)=\sqrt{3} /(2 \beta)$, respectively. Next, as $\alpha=\gamma-1$, the angle of $20^{\circ}$ can be evaluated through the following expressions:

$$
\sin \left(20^{\circ}\right)=\frac{\sqrt{3}}{2 \sqrt{\alpha^{2}+\alpha+1}} \quad \sin \left(20^{\circ}\right)=\frac{\sqrt{3}}{2 \sqrt{\gamma^{2}-\gamma+1}}
$$

An extensive algebraic study of the ratios of the regular nonagon can be found in (Kappraff et al. 2004). In this paper the constants $\alpha, \beta$ and $\gamma$, are determined as limits of the ratios of certain pairs of consecutive terms that are obtained by jumping over generalized Fibonacci sequences. However, because we are more interested in geometric aspects, we focus on a different sequence $c_{n}$ :

$c_{n}: 1,1,1,3,8,23,66,190,547,1575,4535,13058,37599,108262, \ldots$

$$
\begin{aligned}
& c_{n}=3 c_{n-1}-c_{n-3}, n=4,5,6, \ldots \\
& \quad \frac{c_{n+1}}{c_{n}}: \frac{1}{1}, \frac{3}{1}, \frac{4}{3}, \frac{10}{4}, \frac{15}{10}, \ldots \stackrel{n \rightarrow+\infty}{\rightarrow} \gamma=2.87938524 .
\end{aligned}
$$

It is defined by a recurrence relation, such that the ratios of pairs of consecutive terms $c_{n+1} / c_{n}$ converge to $\gamma$, similar to the Fibonacci sequence, where consecutive terms converge to the golden section.
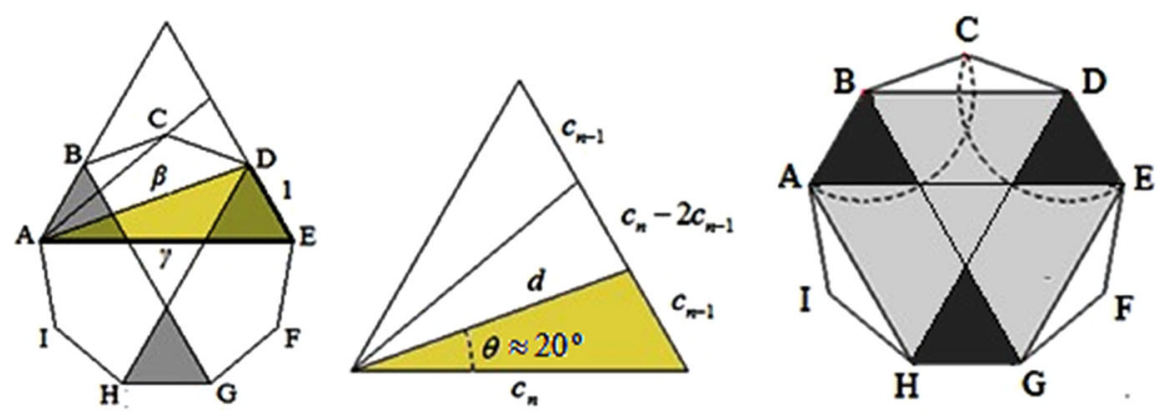

Fig. 15 a Left exact trisection of the $60^{\circ}$ angle and its importance for constructing the regular nonagon; b centre approximated trisection of $60^{\circ}$; c right deriving the equilateral nonagon $A B C D E F G H I$ associated to the hexagon $A B D E G H$ 
Table 1 Successive approximations of $\gamma$ using $c_{n} / c_{n-1}$ and the corresponding approximations of the angle $40^{\circ}$

\begin{tabular}{lll}
\hline$n$ & $c_{n} / c_{n-1}$ & $2 \theta$ \\
\hline 5 & $8 / 3$ & $43.5735785965236^{\circ}$ \\
6 & $23 / 8$ & $40.0679870494440^{\circ}$ \\
7 & $66 / 23$ & $40.1525538863933^{\circ}$ \\
8 & $190 / 66$ & $40.0092482554315^{\circ}$ \\
\hline
\end{tabular}

A Trisection of the $60^{\circ}$ Angle

An accurate classical Greek construction of an angle of $40^{\circ}$ using compass and ruler in a finite steps is impossible. This problem is linked to the classic problem of the trisection of an angle, and here in the particular case of an angle of $60^{\circ}$, and also to methods for solving cubic equations (see Hogendijk 1979 and Özdural 2002). Figure 15a visualizes the equivalence between the construction of the regular nonagon and the trisection of the angle of an equilateral triangle. It is based on the construction of a triangle $A D E$ with angles $20^{\circ}, 60^{\circ}$ and $100^{\circ}$, and with sides of lengths proportional to $1, \beta$ and $\gamma$. We will use consecutive terms of the sequence $c_{n}$ to construct approximations of that triangle.

Figure $15 \mathrm{~b}$ shows an equilateral triangle with side $c_{n}$ dissected into an isosceles triangle with sides $d, d$ and $c_{n}-2 c_{n-1}$, and two triangles with sides $c_{n}, c_{n-1}$ and $d$, and angles $\theta, 60^{\circ}$ and $120^{\circ}-\theta$. Thus, $d^{2}=c_{n-1}^{2}+c_{n}^{2}-2 c_{n-1} c_{n} \cos 60^{\circ}$ and $c_{n-1} / \sin \theta=d / \sin 60^{\circ}$. Therefore

$$
\sin \theta=\frac{\sqrt{3} c_{n-1}}{2 d}=\frac{\sqrt{3} c_{n-1}}{2 \sqrt{c_{n-1}^{2}+c_{n}^{2}-c_{n-1} c_{n}}}=\frac{\sqrt{3}}{2 \sqrt{\left(\frac{c_{n}}{c_{n-1}}\right)^{2}-\left(\frac{c_{n}}{c_{n-1}}\right)+1}} .
$$

Note the similarity between the expressions (1) and (2). We approximated the angle of $20^{\circ}$ by the angle $\theta$, obtained using the constant $\gamma$ and one of the ratios $c_{n} / c_{n-1}$. The terms $c_{n}$ can be expressed in the closed form $c_{n}=A x_{1}^{n}+B x_{2}^{n}+C x_{3}^{n}$, where $A, B$ and $C$ are arbitrary constants, and $x_{1}, x_{2}$ and $x_{3}$ are the solutions of the characteristic equation of the recurrence relation $x^{3}-3 x^{2}+1=0$. Thus, the error of the approximation $\gamma \approx c_{n} / c_{n-1}$ can be evaluated by

$$
\frac{c_{n}}{c_{n-1}}=\frac{A x_{1}^{n}+B x_{2}^{n}+C x_{3}^{n}}{A x_{1}^{n-1}+B x_{2}^{n-1}+C x_{3}^{n-1}} \stackrel{n \rightarrow+\infty}{\rightarrow} \gamma .
$$

Table 1 shows four approximations of the $40^{\circ}$ angle using values of $2 \theta$ obtained from (2).

For instance, taking $c_{5}=8$ and $c_{6}=23$, we get $\theta=20.0339 \ldots{ }^{\circ}$. This implies a division of the side of the triangle into 23 parts and results in a dissection into the parts 8/23, 7/23 and 8/23. The equiangular hexagon shown in Fig. 8 right is determined by its triangular skeleton and the equiangular hexagon with sides 8 and diagonals 23 will result in a derived equilateral nonagon with sides 8 and third diagonal 23 . The three central angles will be $2 \theta=40.0679 \ldots{ }^{\circ}$, alternating with two 


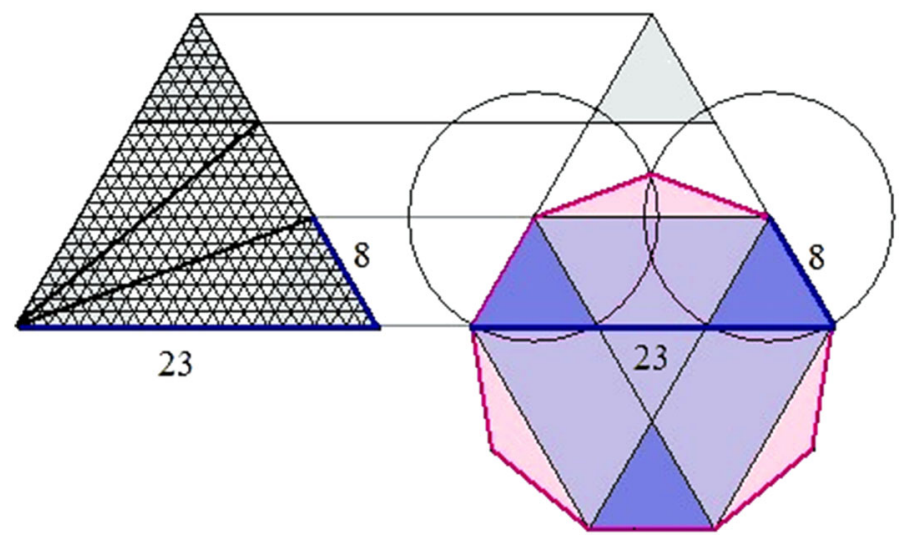

Fig. 16 An almost regular nonagon obtained from the dissection 8-7-8 of the side of the equilateral triangle

angles of $60^{\circ}-\theta$. The dissection $8 / 23-7 / 23-8 / 23$ produces nonagons that are perceived as regular by the naked eye (Fig. 16).

From the eleventh century, several methods for approximating a construction of the regular nonagon are known in Islamic architecture. From the elements of the patterns with hexagons and nonagons, one can guess that the craftsmen applied an approximated solution of the trisection of the angle of $60^{\circ}$, consciously or not. However, we have to keep in mind that skilful craftsmen did not make the patterns by means of quotients of consecutive terms of recurrence sequences, nor by exact mathematical constructions, but using practical methods. The lack of accuracy in the geometric construction is obvious in the stucco marble frieze of the Mahfil in Hagia Sophia (Fig. 3) and in the panel of its minbar (Fig. 5). In contrast, the woodwork decoration of the minbar of Selimiye Mosque looks almost perfect (Fig. 2). We will see that the concept of the pattern seems to be the same and so perhaps the difference in the result can be explained by the materials used to execute them (wood, stucco, stone, etc.).

The mutual influence of the methods of mathematicians and craftsmen is wellknown. There are several Persian medieval treatises about the 'conversazioni' between both groups (see, for example, Hogendijk 1979; Özdural 1996, 2002). Yet, the texts provide little information about what exactly the craftsmen's method was. For instance, the construction of the nonagon is explained in these treatises without saying how it was actually done, probably because the better construction methods required solving a cubic equation or studying conics. Perhaps these topics were too advanced for craftsmen, who preferred methods like trial-error or dissection and composition. Thus, in view of the practical application, we searched for angles of about $20^{\circ}$ in the shapes composed by simple polygons as the equilateral triangle, the square, the regular hexagon, the octagon and the dodecagon. Simple procedures for obtaining angles close to $40^{\circ}$ could have been useful for craftsmen to realize the nonagonal geometric patterns. 

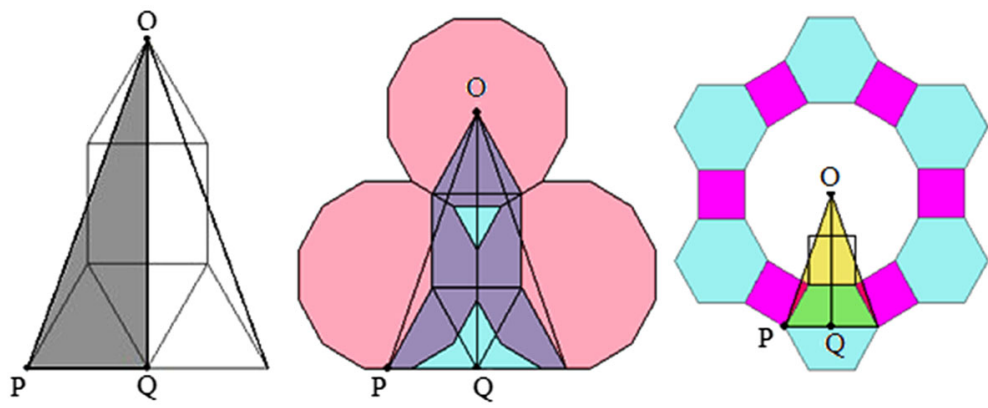

Fig. 17 a Left the angle of $20.1^{\circ}$ from a square and three triangles; b, centre and c, right the $20.1^{\circ}$ angle on two semi-regular tessellations
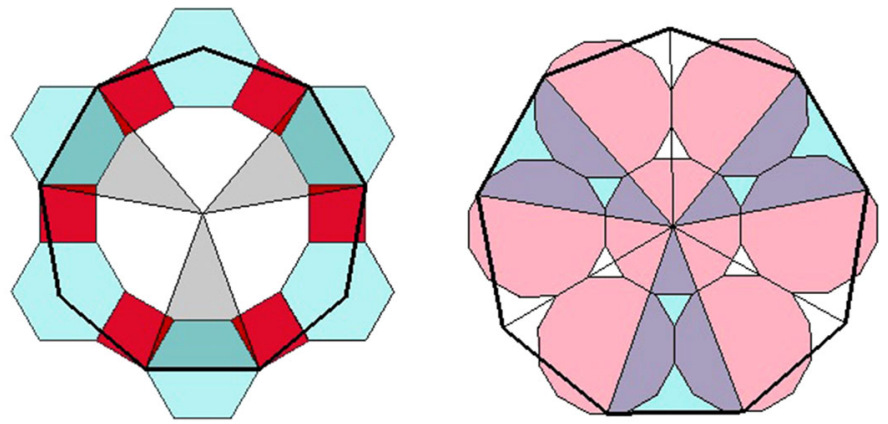

Fig. 18 Nonagons with angles $40.2^{\circ}-39.9^{\circ}-39.9^{\circ}$ overlap two semi-regular tessellations

Approximations of the $20^{\circ}$ Angle

The $20.1^{\circ}$ Angle, Starting from a Square and an Equilateral Triangle or a Regular Hexagon

The shape shown in Fig. 17a is composed, from top to bottom, by an equilateral triangle, a square and one half of hexagon, that is, three equilateral triangles. Hence the angle $\angle P O Q$ is equal to $\operatorname{arctg}(1 /(\sqrt{3}+1)) \approx 20.1^{\circ}$.

The angles of $60^{\circ}$ and $90^{\circ}$ in triangle $P O Q$ imply the angle $20.1^{\circ}$ can be found in some semi-regular tessellations composed of hexagons, dodecagons, squares and triangles. Figure 17 shows the triangle $P O Q$ overlapping two semi-regular tessellations and Fig. 18 the nonagons determined in the corresponding tessellations.

The $20.1^{\circ}$ Angle, Starting from a Regular Octagon and a Dodecagon

The drawing in Fig. 19a is obtained as follows:

1. Draw a regular dodecagon and a chord of $90^{\circ}, A B$. Call its centre $O$.

2. Draw a regular octagon such that $A B$ is a chord of $90^{\circ}$. Call its centre $P$. 

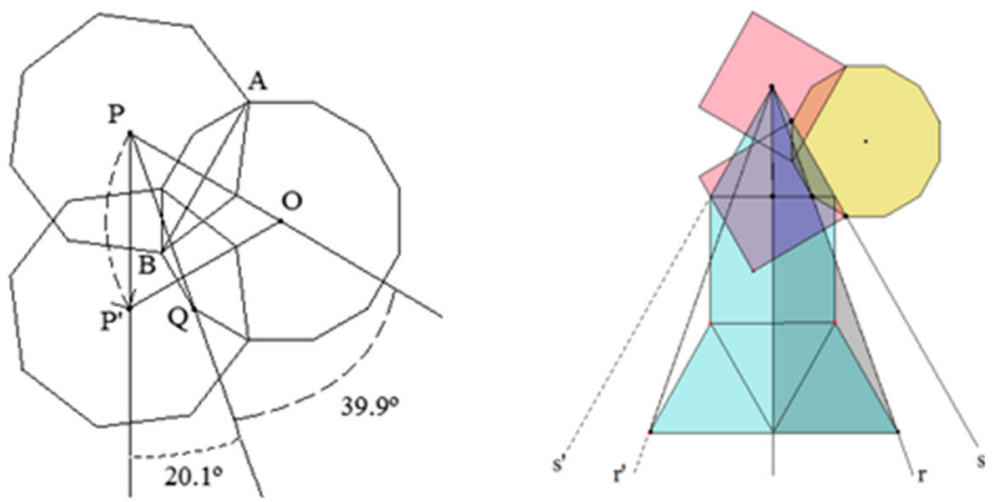

Fig. 19 a Left construction of an angle of $20.1^{\circ}$ using an octagon and a dodecagon; $\mathbf{b}$ right proof that the construction given in (a) is equivalent to the construction of Fig. 17
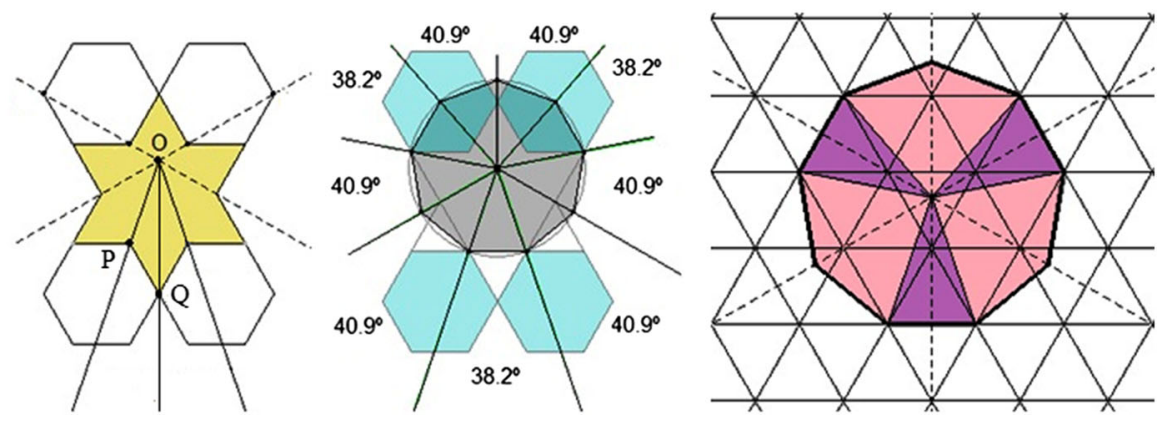

Fig. 20 a left the $38.2^{\circ}$ angle based on a hexagon and a six pointed star; b centre a nonagon with angles $38.2^{\circ}$ and $40.9^{\circ}$; c right the same nonagon on a triangular net

3. Rotate the octagon over $60^{\circ}$ around the point $O$ so that $P$ is mapped onto $P$ '.

4. Call $Q$ the vertex in the dodecagon such that the chord $A O$ is $120^{\circ}$.

5. Drawing the segments $P O, P Q$ and $P^{\prime}$; then $\angle O P Q \approx 39.9^{\circ}$ and $\angle Q P P I \approx 20.1^{\circ}$.

Using dynamic geometry software (such as Geogebra or Cabri) we can check in Islamic style, that is, by moving geometric shapes, that both preceding approximations of $20.1^{\circ}$ are identical (Fig. 19b).

The $19.1^{\circ}$ Angle

The tessellation by hexagons and equilateral triangles is especially interesting. Figure 20 a determines the angle $\angle P O Q=\arccos (11 / 14) \approx 38.2^{\circ}$ providing the approximation $19.1^{\circ}$ for the $20^{\circ}$ angle. This again produces an apparently regular nonagon in a very simple way (Fig. 20b). The angles of $19.1^{\circ}$ and $38.2^{\circ}$ can be constructed by just one single triangular net (Fig. 20c) and will play an important role in one of the proposed practical construction of the patterns. Note that the 

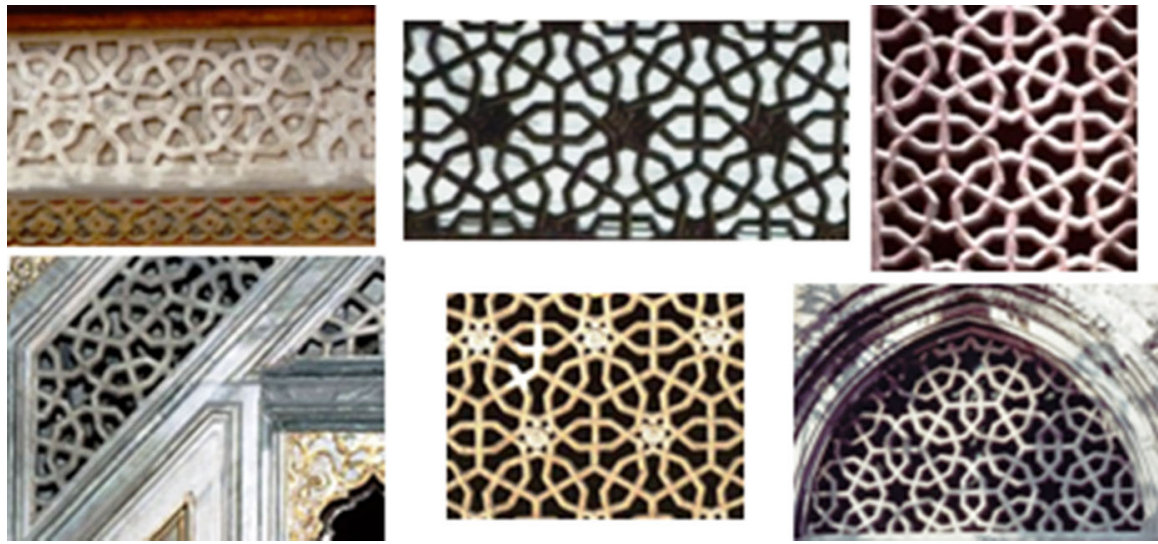

Fig. 21 Patterns found in the examples already considered

nonagon obtained from this method has its centre on the vertex point of $\angle P O Q$ which is located in the centre of one of the equilateral triangles of the net.

Figure 21 summarizes some examples where a rosette is seen formed by six overlapping nonagons centred in the vertices of a hexagonal grid. Each of the elementary rosettes in these interlocked patterns can be thought of as generated by a nonagon that turns over $60^{\circ}$ five successive times with respect to the centre of the hexagons of the grid, after decreasing the side of the hexagons of the grid.

Different ratios $r$ between the side of the hexagon and the nonagon lead to different variations of the pattern that we call pattern $p(r)$. They have different visual aspects, but are geometrically equivalent. Figure 22 shows some patterns belonging on the family of patterns $\{p(r): 0<r<+\infty\}$, ordered by decreasing ratios.

Note that the overlapping of the nonagons doesn't happen when $\gamma \geq \sqrt{3} \alpha \beta / 3 \approx 2.75$, and the central star of the rosette becomes like a six-petal shape if $\gamma<\sqrt{3} /\left(6 \sin 10^{\circ}\right) \approx 1.66$. Thus, we will only consider patterns $p(r)$ such that $\sqrt{3} /\left(6 \sin 10^{\circ}\right) \leq \gamma<\sqrt{3} \alpha \beta / 3$. Assuming that the nonagons are regular, we will analyse the theoretical pattern $p(r)$ from different points of view. To make it easier, we again suppose that the length of the side of the nonagon is 1 and the length of side of the hexagon is $r$.

Geometric Analysis of the General Family of Patterns $p(r)$

The interlaced nonagons and hexagons determine a tiling (for the initiated, it is of type p6 m (*632); see for instance Grünbaum and Shepard 1987) that can be obtained by edge to edge union of three different components or elementary tiles: I, an equilateral six-pointed star; II, an irregular convex pentagon we will call house; III, an irregular concave octagon we will call bow (see Fig. 23).

Some observations concerning the triangle $F G O$ in Fig. 23: 


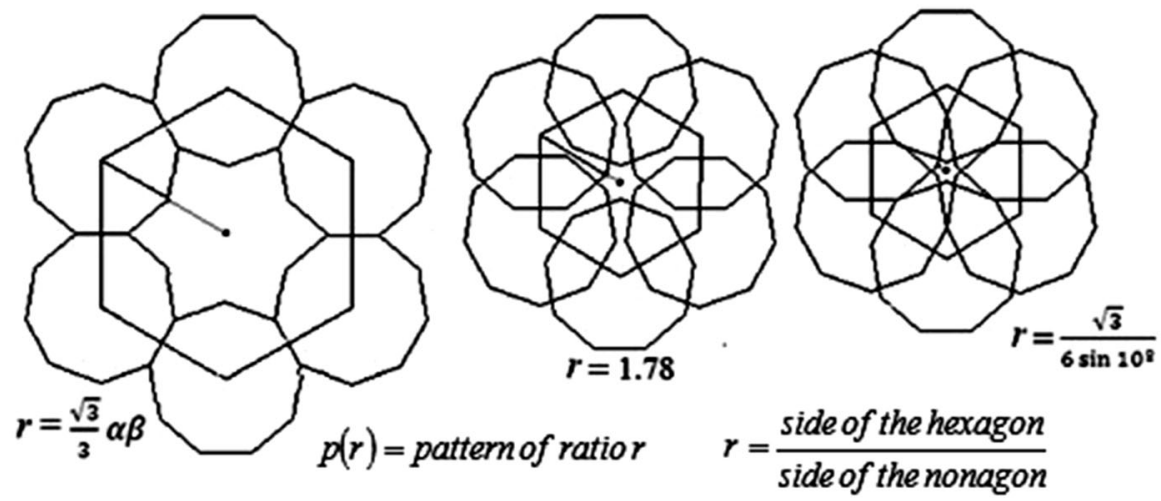

Fig. 22 Particular cases of the pattern $p(r)$
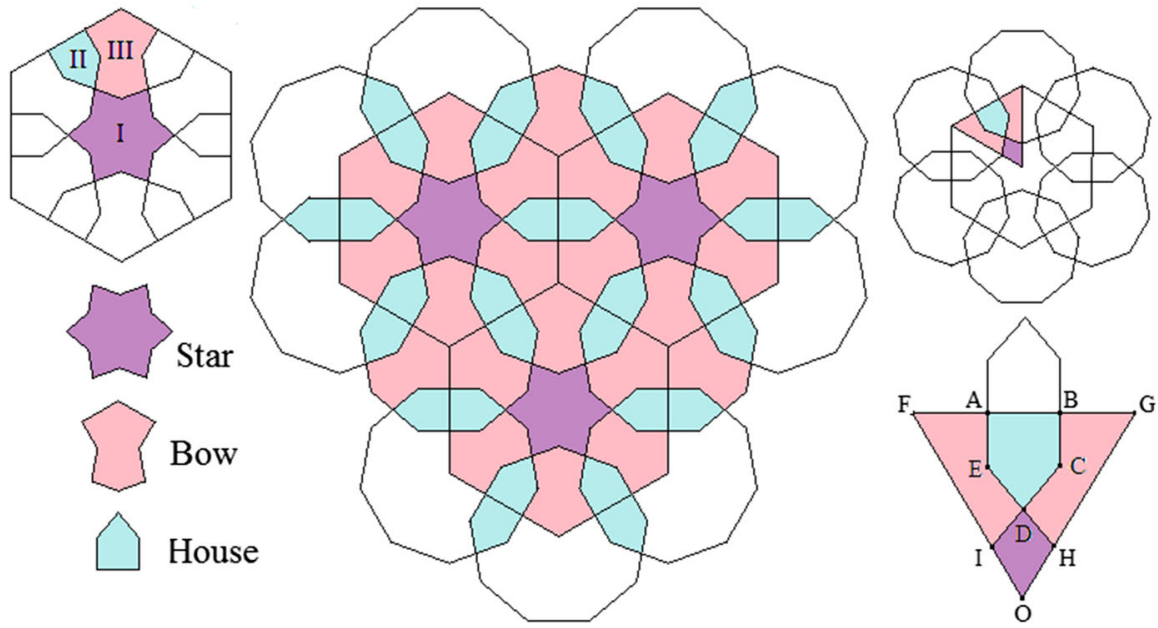

Fig. 23 Elementary tiles I, II and III and basic polygon FGO

1. The side of the nonagon has length 1 . The side of the hexagon has length $r$, and is divided in three line segments $F A, A B$ and $B G ; B C=1 / 2$.

2. The sides of the hexagon and the nonagons are perpendicular in their intersections $A$ and $B$. The points $E$ and $C$ are vertices of the regular nonagons, and thus

$$
\angle B C D=\angle D E A=140^{\circ} \text { and } \angle C D E=\angle H D I=80^{\circ}
$$

3. $F B$ is the apothem of the nonagon, so

$$
F B=\frac{1}{2 \tan 20^{\circ}} \quad F A=B G=r-\frac{1}{2 \tan 20^{\circ}} \quad A B=E C=\frac{1}{\tan 20^{\circ}}-r
$$




$$
E D=D C=\left(\frac{1}{\tan 20^{\circ}}-r\right) \frac{\sin 50^{\circ}}{\sin 80^{\circ}}=\frac{\left(1-r \tan 20^{\circ}\right)}{4 \sin ^{2} 20^{\circ}}
$$

Note that the length of the perimeter of the tiles I, II and III, depends on the value of the parameter $r$ for given a pattern $p(r)$, though the angles of the tiles are constant. The angles in the vertices of the star tile I are $80^{\circ}$ and $220^{\circ}$; the angles of the house tile II are $80^{\circ}, 140^{\circ}$ and $90^{\circ}$; and the bow tile III has angles of $120^{\circ}, 90^{\circ}, 220^{\circ}, 100^{\circ}$ and $140^{\circ}$.

The pattern can also be considered as the superposition of three different layers, $\mathrm{L}_{1}, \mathrm{~L}_{2}$ and $\mathrm{L}_{3}$ (Fig. 24). Layer $\mathrm{L}_{1}$ is a hexagonal net of side $r$ while layers $\mathrm{L}_{2}$ and $\mathrm{L}_{3}$ are formed by regular nonagons with centres on the vertices of an imaginary equilateral triangular grid with side $\sqrt{3} r$. Both layers of nonagons $\mathrm{L}_{2}$ and $\mathrm{L}_{3}$ coincide under translation by vectors $\mathrm{v}_{1}, \mathrm{v}_{2}$ and $\mathrm{v}_{3}$ (Fig. 24).

Intersection, union and/or subtraction of the layers $\mathrm{L}_{1}, \mathrm{~L}_{2}$ and $\mathrm{L}_{3}$ determines different layers $\mathrm{L}_{4}, \mathrm{~L}_{5}$ and $\mathrm{L}_{6}$ (Fig. 25a), generating the same pattern. Each pattern $p(r)$ can also be generated by edge to edge addition of a basic polygon (a shape that covers the tiling using only isometries). Figure 23 showed one of them, but the smallest polygon with this property is the right triangle $\mathrm{V}$ with angles $30^{\circ}, 60^{\circ}$ and $90^{\circ}$ and determined by the radius of the hexagon, the apothem of the nonagon and the half-side of the hexagon between them (Fig. 25b). This small shape covers the tilling by reflections only. We can also find a rectangular basic polygon, such as VI. We call these polygons V and VI practical tiles, because their triangular or rectangular shape makes it possible to use them as a template for a practical construction, especially for friezes.

\section{Geometrical Analysis of the Practical Patterns}

In the practical realizations of the pattern in the grilles, panels and friezes given in the examples, the nonagons are perceived as regular. However, one can wonder if
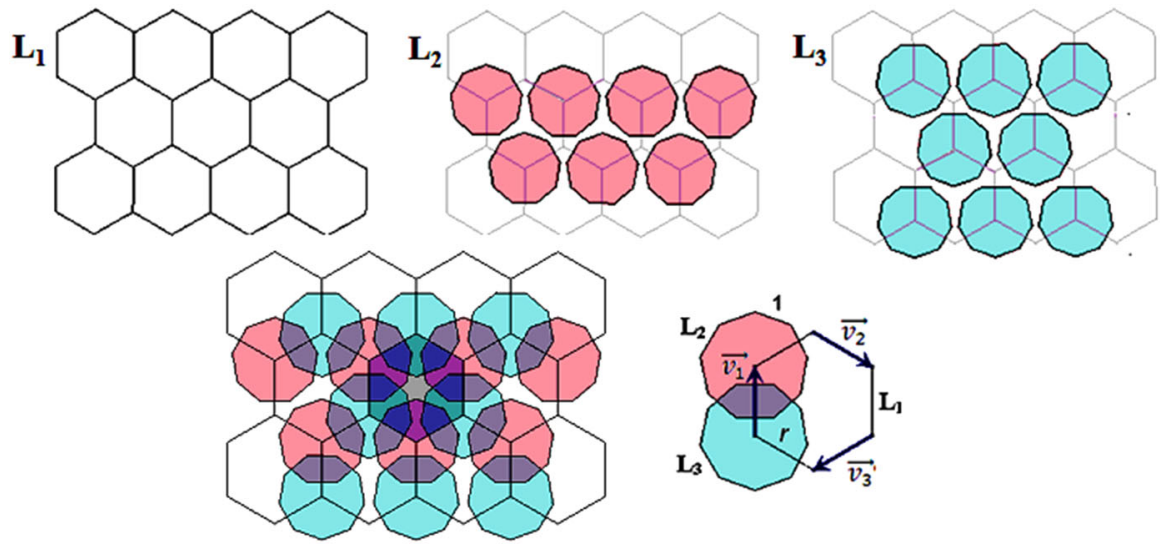

Fig. 24 The hexagonal layer $\mathrm{L}_{1}$, the nonagonal layers $\mathrm{L}_{2}$ and $\mathrm{L}_{3}$, and how they come together 

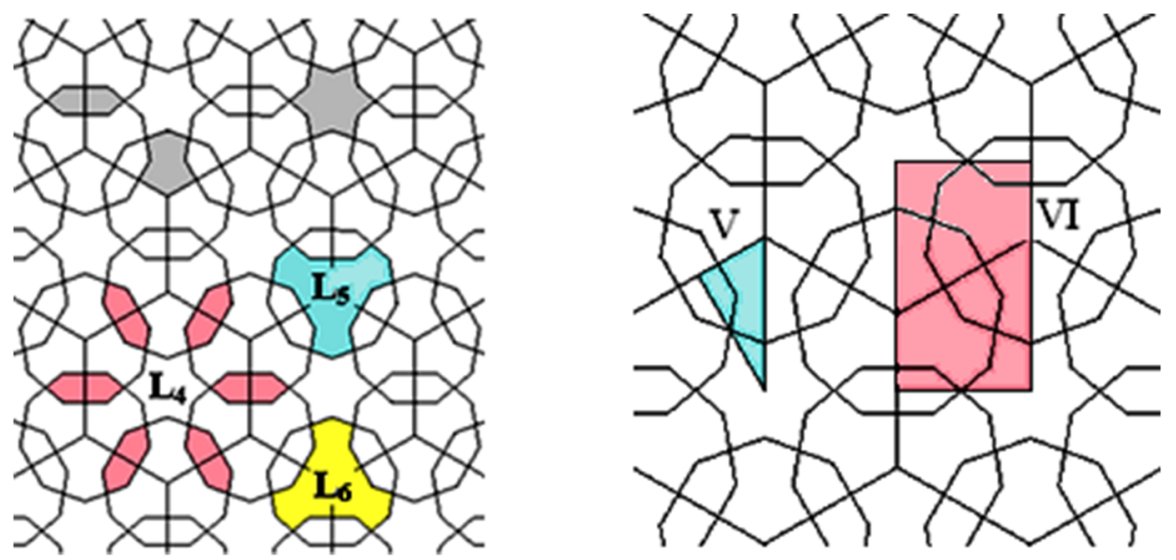

Fig. 25 a Left the sub-unities $\mathrm{L}_{4}, \mathrm{~L}_{5}$ and $\mathrm{L}_{6}$; $\mathbf{b}$ right the smaller basic polygon $\mathrm{V}$ and the smaller practical tile VI

the nonagons were regular in the mind of the designer: what could their mathematical foundations have been? We will not need measurements, because careful observation of the patterns gives theoretical arguments suggesting the designed nonagons are necessarily non-regular.

Figure 26 shows that the extension of one of the radii of the nonagons intercepts the side of the adjacent hexagon (of which one vertex is the centre of the nonagon) in the midpoint. This observation is emphasized using overlapping diagrams.

This simple test is valid even with the usual problem of the perspective distortion of the images, because all the designs are in the plane. If we consider the regular hexagon of Fig. 27, the shaded triangle will have an angle of $120^{\circ}$ between the sides $a$ and $a / 2$.

Thus:

$$
d=\frac{\sqrt{7} a}{2} \quad \sin \theta=\frac{\sqrt{3}}{2 \sqrt{7}}=\frac{\sqrt{21}}{14} \quad \theta=\arcsin \frac{\sqrt{21}}{14} \approx 19.1^{\circ} \Rightarrow 2 \theta \approx 38.2^{\circ}
$$

This implies that the angles of the nonagons of Selimiye Mosque and Hagia Sophia are $2 \theta \approx 38.22^{\circ}$ and $60^{\circ}-\theta \approx 40.89^{\circ}$. The same angle was obtained in Fig. 20 by a semi-regular tessellation.

As for the nonagon and the hexagon displayed in Fig. 28, the extension of the radius of the nonagon intersects the side of the hexagon at the midpoint and if this extension is prolonged beyond the midpoint, a vertex of a hexagon is obtained.

Figure 28 presents the parallelogram $O A B C$ and the midpoint $M$ where the diagonals $O B$ and $A C$ intersect $F D$. It follows that

$$
\mathrm{OB}=\sqrt{7} \text { and } \sin \theta=\frac{\sin 120^{\circ}}{\sqrt{7}}=\frac{\sqrt{21}}{14} \Rightarrow \theta=\arcsin \left(\frac{\sqrt{21}}{14}\right) \approx 19.1^{\circ}
$$

Note that, in spite of the different locations of the nonagon and the hexagon in Figs. 27 and 28, the calculations are indeed the same, and they twice yield an angle 

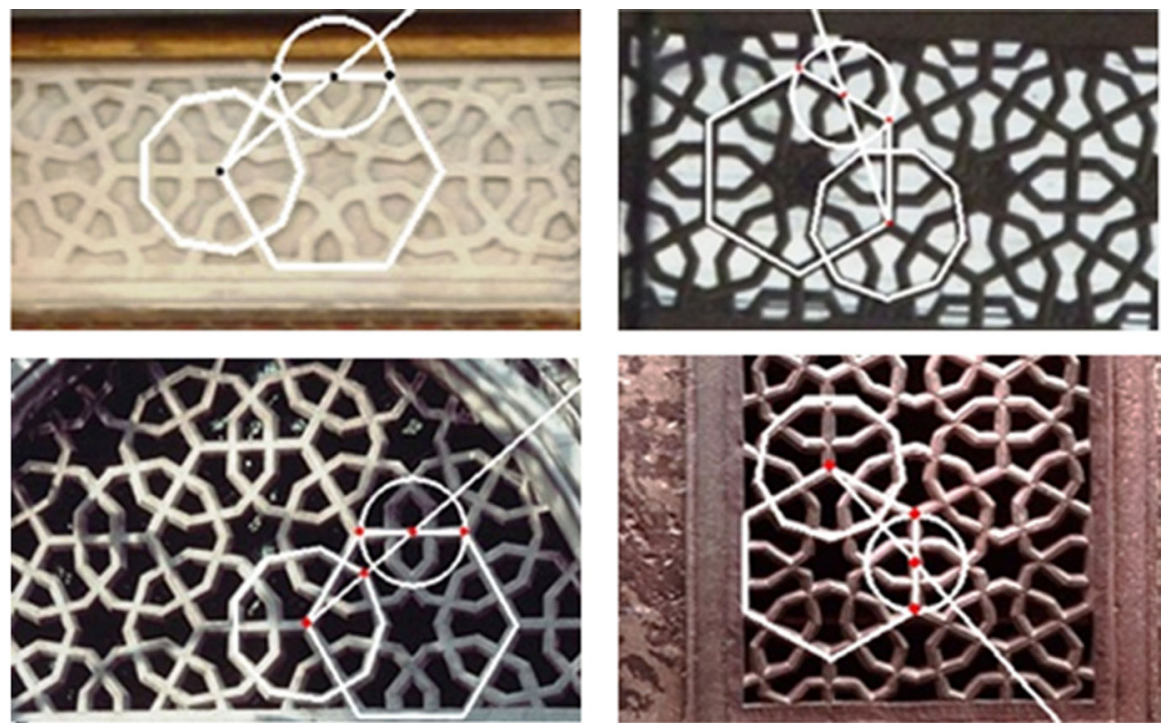

Fig. 26 Extending the radius of the nonagon intersects the side of a hexagon in the midpoint
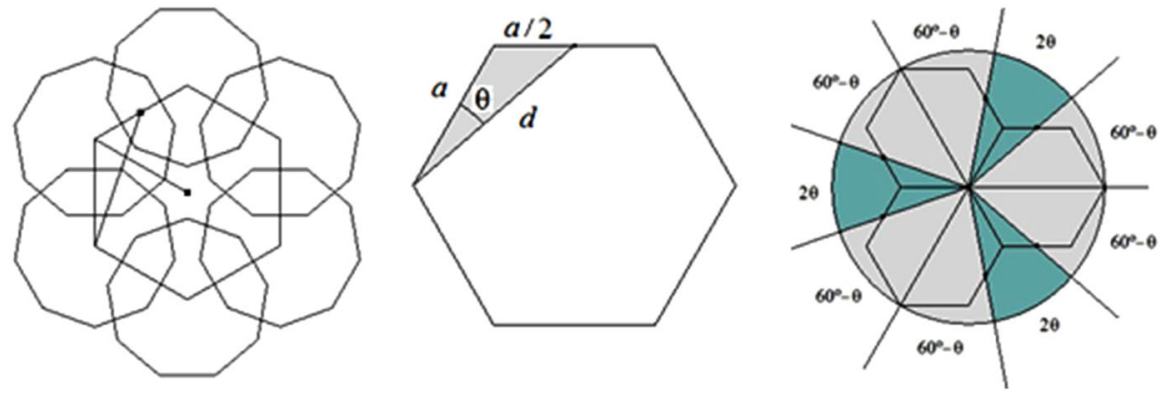

Fig. 27 The symmetry of the pattern and the midpoint property

of $19.1^{\circ}$ angle. Both graphical properties occur in all of the analyzed practical patterns. There is thus a very simple way to construct the angles of the nonagons of all of the examples. We apply the two preceding observations to the theoretical pattern $p(r)$, where the nonagons are perfectly regular.

The nonagon in Fig. 29 is regular, but a simple glance shows the point $B$ is not the midpoint of the side $A C$ of the hexagon. Indeed, the three rectangles $O A B, D F G$ and $G F H$ have angles $20^{\circ}, 120^{\circ}$ and $40^{\circ}$, and thus they are similar; therefore

$$
\frac{A C}{A B}=\frac{O A}{A B}=\frac{D F}{F G}=\frac{\text { Fist diagonal of the nonagon }}{\text { side of the nonagon }}=\alpha \approx 1.88,
$$

that is, the ratio $A C / A B$ is constant in all $p(r)$ patterns and equals $\alpha$, not 2 .

Figure 30 visualizes the second observation. Again, it turns out to be only an approximation when checked out in the theoretical patterns. 

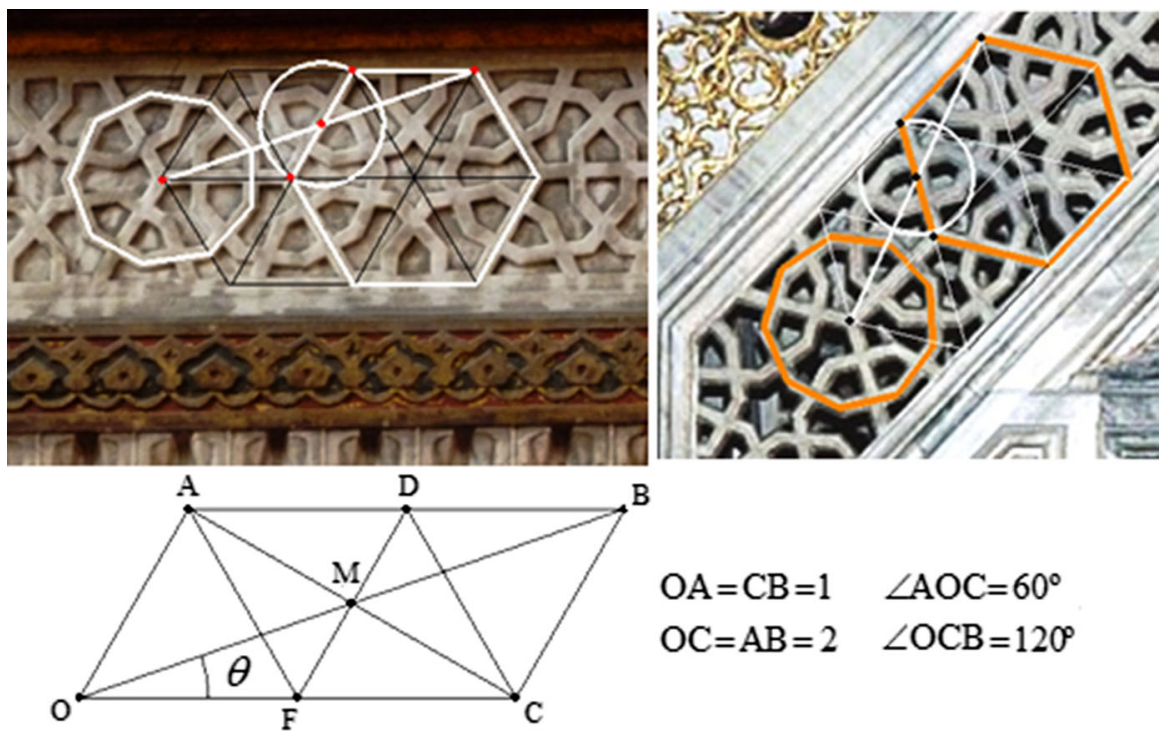

$$
\begin{array}{ll}
\mathrm{OA}=\mathrm{CB}=1 & \angle \mathrm{AOC}=60^{\circ} \\
\mathrm{OC}=\mathrm{AB}=2 & \angle \mathrm{OCB}=120^{\circ}
\end{array}
$$

Fig. 28 Above diagrams and details of frieze in the Hagia Sophia (left) and the Selimiye Mosque (right); below drawing showing the line segment $O B$, where $O$ is the center of the nonagon, $B$ is the vertex of the hexagon centered in $C$ and $M$ is the midpoint of $F D$ and $O B$

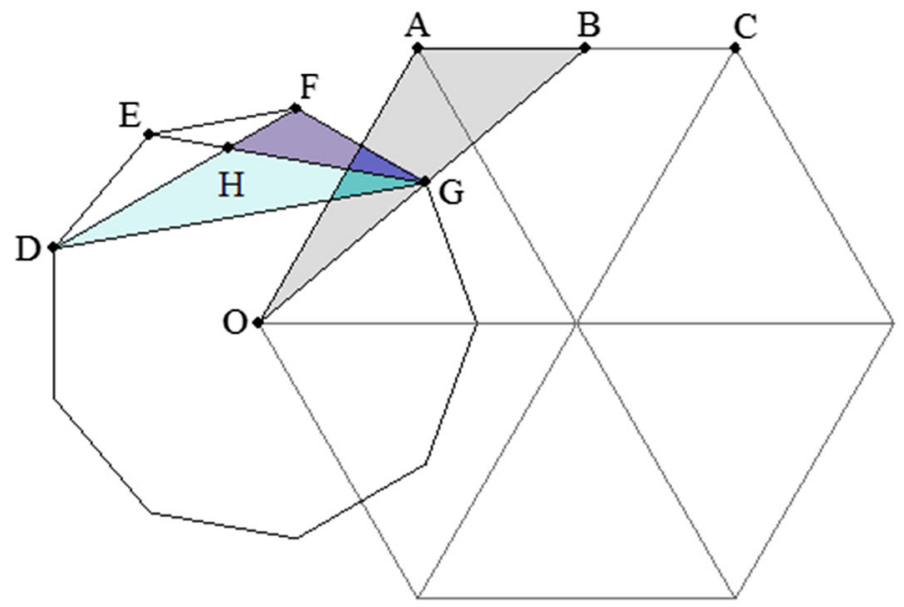

Fig. 29 Property of the regular nonagon centered in the vertex of the regular hexagon

For a regular nonagon, $\angle P O E=20^{\circ}$ and this is greater than $\theta$. Consequently, the radius $O P$ of the nonagon does not lie on the diagonal $O B$, and thus the points $R$ and $B$ are distinct points. Therefore, the construction of the pattern by using exact regular nonagons is not possible.

Since in all the cases the angles of the practical patterns are the same, the slight visual differences among the different analyzed patterns are consequences of the 


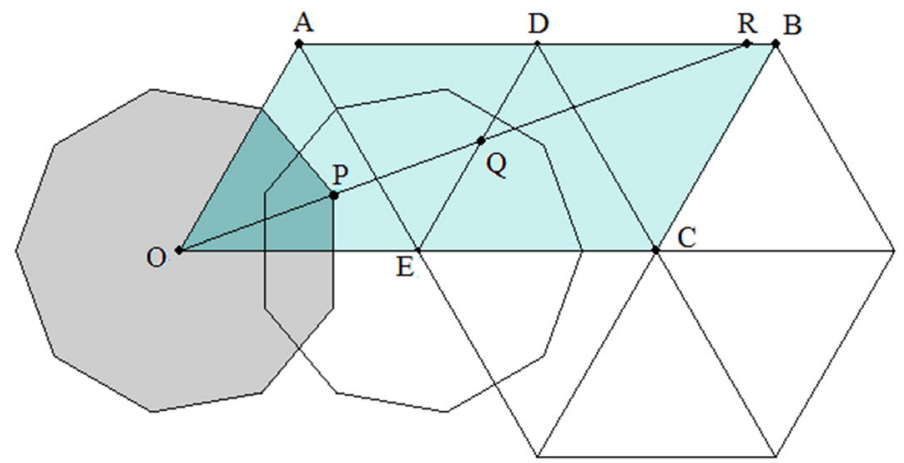

Fig. 30 The extension of the radius $O P$ of the regular nonagon doesn't pass through vertex $B$
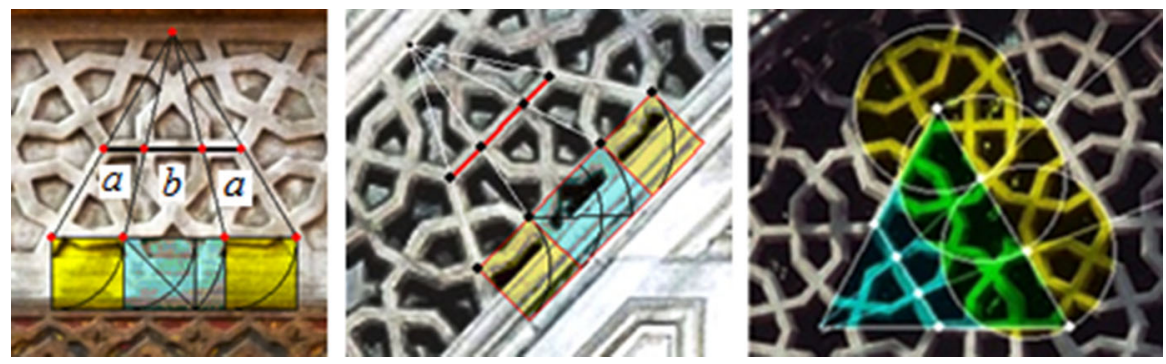

Fig. 31 Graphic checks to verify that ratios $b / a$ are around 1.4 in the Hagia Sophia (left) and the Selimiye Mosque (middle); and b/a around 1 in the Mausoleum of Shehzade Mehmet (right)

different ratios between the side of the hexagon and the side of the nonagon. This can be estimated by the ratios of the three segments obtained from the intercepting points of the polygons of the grid, as in Fig. 31. If call $a-b-a$ the lengths of the three parts determined in the side of the hexagon, we observe a ratio $b / a$ around 1.4 $\approx \sqrt{2}$, in the Hagia Sophia and the Selimiye Mosque. However, in the grill of the window of the Mausoleum of Shehzade Mehmet this ratio is approximately 1, while it is close to $2 / 3$ in some similar modern screens.

Finally, according the two preceding observations from Figs. 26 and 27, we construct the pattern of the frieze of the mahfil in the Hagia Sophia, and we provide a second method to construct the pattern.

Two Constructions for a Pattern in Hagia Sophia

\section{Construction 1}

The angles of the nonagons of a frieze in Hagia Sophia have been already determined. Thus, in the first step we set out these central angles over a hexagonal net in the way shown in Fig. 27 (right), and so the nonagon is determined for a given radius. We observe that one of the vertices of the nonagon, $P$, is the intersection of 
the line segment $A B$ and the line passing by $O$ and $C$ (Fig. 32). We locate the points $A, B, C$ in a Cartesian coordinate system with origin in the center of the nonagon. The $x$-axis is the line containing $O B$, and the side of the hexagon is taken as unit length.

In this reference system, the coordinates of the considered points are $O(0,0)$, $A(-3 / 2, \sqrt{3} / 2), B(6,0)$ and $C(1, \sqrt{3})$. Since the segments $A B$ and $O C$ concur in the point $P$, it follows that

$$
\begin{aligned}
P(\lambda, \sqrt{3} \lambda) & =P(6+15 \mu,-\sqrt{3} \mu) \Rightarrow \lambda=-\mu=\frac{3}{8} \Rightarrow P\left(\frac{3}{8}, \frac{3 \sqrt{7}}{8}\right) \\
& \Rightarrow O P=\sqrt{\left(\frac{3}{8}\right)^{2}+\left(\frac{3 \sqrt{7}}{8}\right)^{2}}=\frac{3}{4}
\end{aligned}
$$

Thus, point $P$ leads to a very easy value of the radius, 3/4, which is three-quarters of the side of the hexagon. Figure 33 visualizes the construction of the nonagon.

Because of the rotational symmetry, the rosette can be completed by successive rotation over $60^{\circ}$ of the nonagon with respect to the center of the nearby hexagon (Fig. 34). A translation of the rosette by the vector $v=(3,0)$ completes the frieze.

The nonagons of this construction have three sides with length $l_{1}=3 \sqrt{21} / 28$. This value of the sides of the nonagon produces dissections $a-b-a$ of the side of hexagon with ratio $b / a=(30 \sqrt{7}-56) /(56-15 \sqrt{7})=1.432 \ldots$ This approximation corresponds to the graphical estimations of Fig. 31.
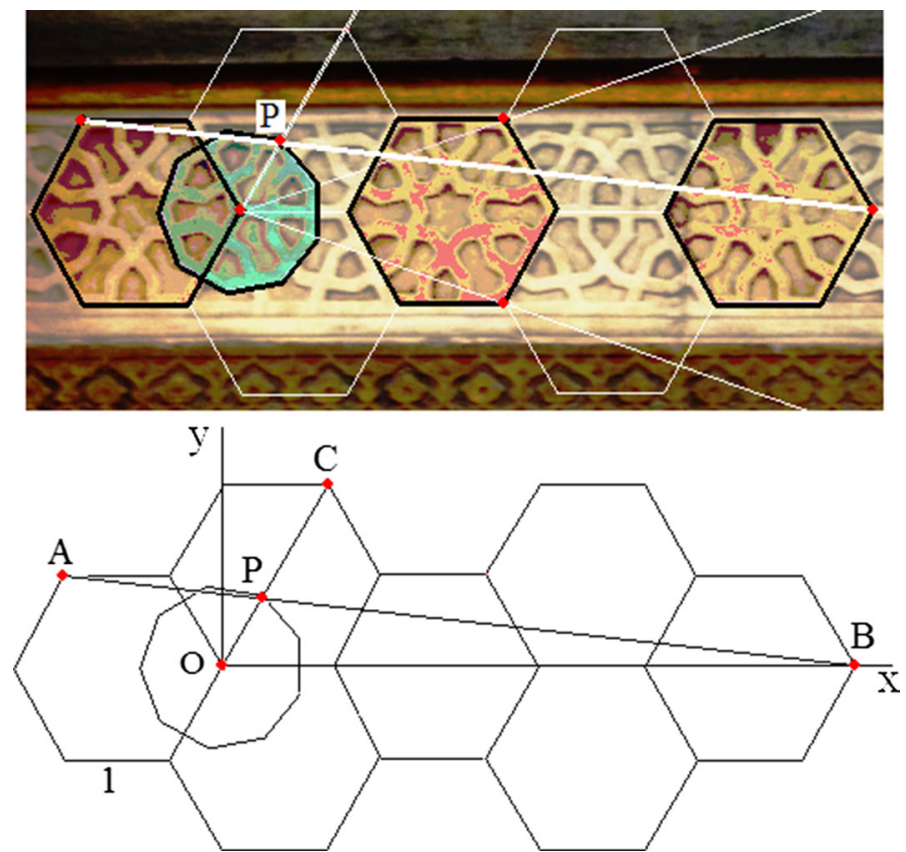

Fig. 32 Nonagon of the frieze with three angles of $38.2^{\circ}$, six angles of $40.9^{\circ}$, and radius $O P$ 


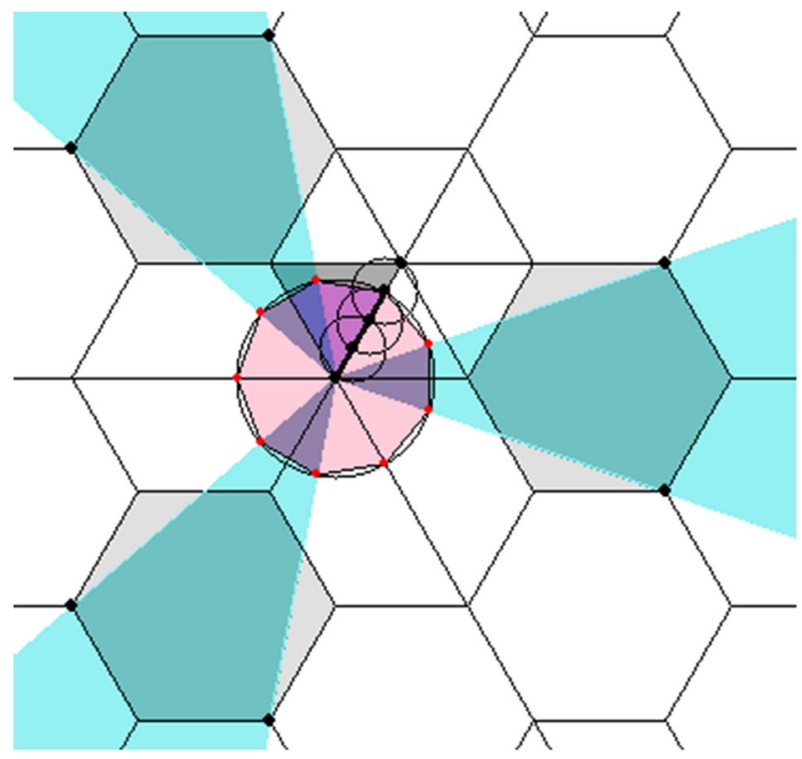

Fig. 33 Construction of a nonagon with three angles of $38.2^{\circ}$, six angles of $40.9^{\circ}$ and a radius that is $3 / 4$ of the hexagonal net with side 1

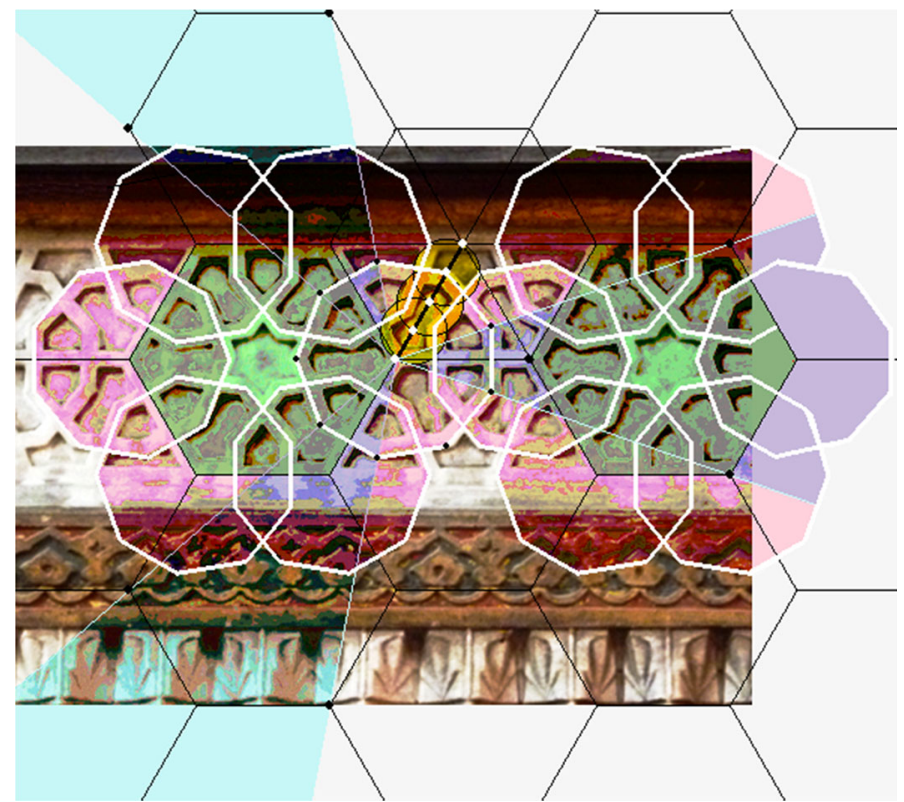

Fig. 34 A geometric construction and the real frieze 


\section{Construction 2}

The geometric analysis of the theoretical pattern given above guarantees that the grid of interlocking hexagons and nonagons can be constructed using the basic polygon $\mathrm{V}$ shown in Fig. 25 or from the triangle $F G O$ defined in Fig. 23. However, it can also be derived based only on the house tile II, over a hexagonal net. Figure 35 shows the sides $E B$ and $E F$ produce the two sides of the irregular nonagon. The angles $\theta$ and $\angle E F G=360^{\circ}-2 \theta$ determine six angles $\theta$ and three angles $\alpha$ in the nonagon, in the order $\theta-\theta-\alpha$.

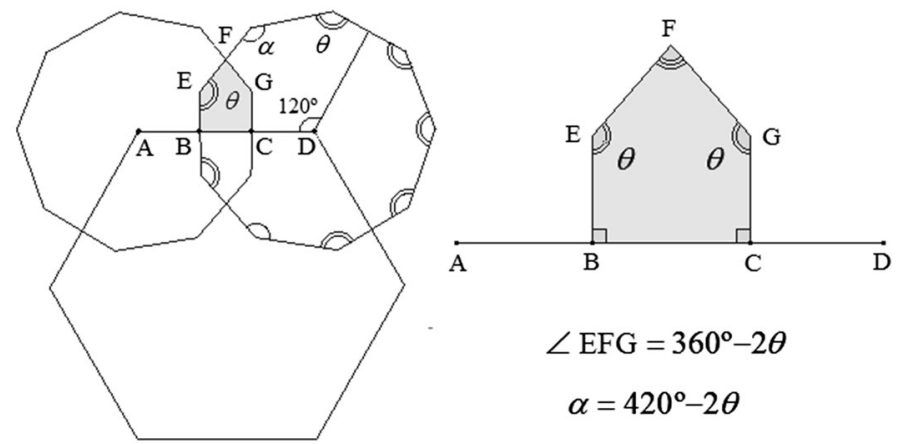

Fig. 35 The angles of the house tile determine the angles of the nonagons
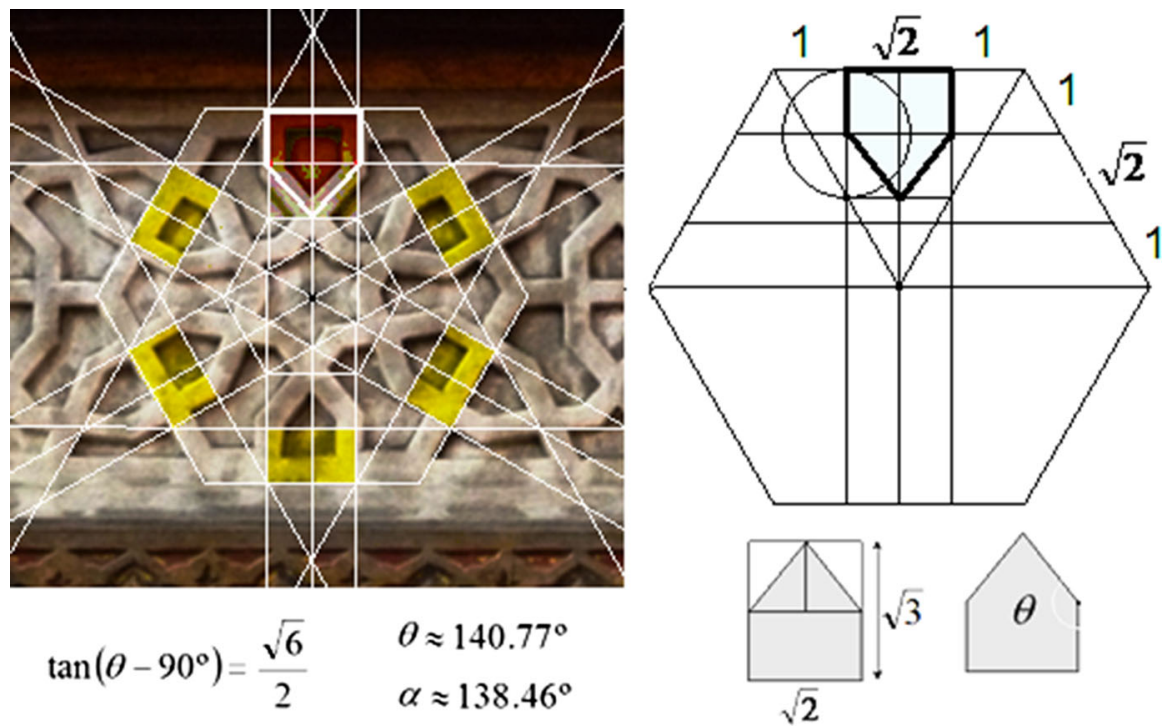

Fig. 36 House tile for the frieze of Hagia Sophia 

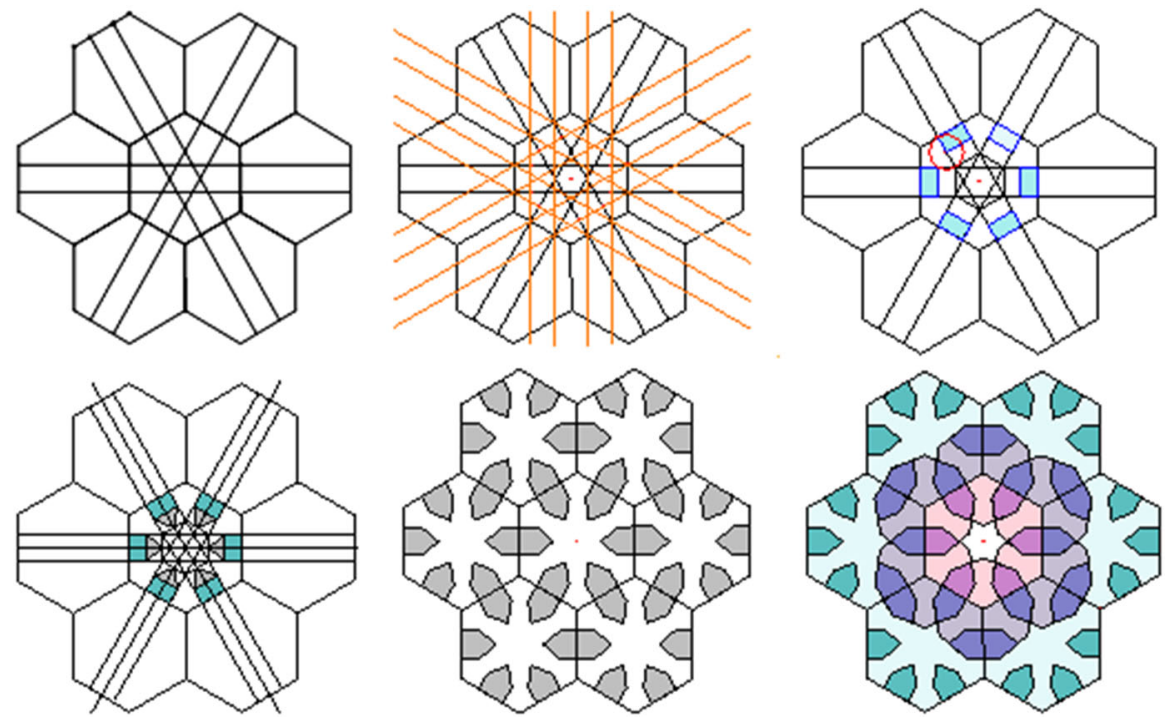

Fig. 37 Steps for the construction of the rosette starting from the house tile of Fig. 36

The analysis of Fig. 31 suggest a hypothetic ideal ratio $b / a \approx \sqrt{2}$, and thus we assume that the nonagons dissect the side of the hexagon in three segments $A B, B C$ and $C D$ such that $A B=C D$ and with ratio $B C / A B=\sqrt{2}$. This choice allows us a simple way to construct the house tile and complete pattern by intersection of straight lines in a hexagonal net.

Figure 36 shows the house tile as the union of a rectangle with ratio $2 \sqrt{6} / 3$ and an isosceles triangle inscribed in another rectangle with the same ratio. Note that $2 \sqrt{6} / 3 \approx 1.6$ is an good approximation of the golden section, and thus the pattern of Hagia Sophia and Selimiye Mosque constructed in this way almost looks as if it respects the golden section proportion. Figure 37 explains the successive steps to construct a rosette of the pattern.

Of course, we don't know the exact procedure of the craftsmen who constructed the nonagonal patterns on Hagia Sophia and the Selimiye Mosque, but the hypothesis of a construction in a way similar to our Construction 1 seems quite realistic.

\section{References}

Al-Biruni. 1954-1956 A.D./1373-1375 A.H, Al-Qanunu'l-Mas'udi (Canon Masudicus). Hyderabad: Osmania Oriental Publication Bureau. 3 vols.

Grünbaum, Branko, Shepard, Geoffrey C. 1987. Tilings and Patterns. New York: W.H. Freeman

Hogendijk, Jan P. 1979. On the trisection of an angle and the construction of a regular nonagon by means of conic sections in medieval Islamic geometry, unpublished ms., Preprint 113, March 1979, 
University of Utrecht, Department of Mathematics. Retrieved from http://www.jphogendijk.nl/publ. html, 19 Dec. 2014.

Kappraff, Jay, Jablan, Slavik, Adamson, Gary W. and Sazdanovic, Radmila. 2004. Golden Fields, Generalized Fibonacci Sequences, and Chaotic Matrices. Forma 19: 367-387.

Özdural, A. 1996. On Interlocking Similar or Corresponding Figures and Ornamental Patterns of Cubic Equations.Muqarnas13: 191-211.

Özdural, A. 2002. The Use of Cubic Equations in Islamic Art and Architecture. pp. 165-179 in Nexus IV: Architecture and Mathematics, Kim Williams and Jose Francisco Rodrigues, eds. Fucecchio (Florence): Kim Williams Books. Rpt. in Architecture and Mathematics from Antiquity to the Future, vol. I, Kim Williams and Michael J. Ostwald, eds., Cham, Springer, 2014, pp. 467-482. Steinbach, Peter. 1997. Golden Fields: A Case for the Heptagon. Mathematics Magazine 70: 22-31.

Antonia Redondo Buitrago has a $\mathrm{PhD}$ in applied mathematics from the University of Valencia, Spain. Her research interests are fractional powers of operators, continued fractions, the relevant proportions (plastic number, Cordovan proportion, meta-golden ratio and metallic means) and non-constructible polygons. Her contributions in the field of geometry are mainly focused on the interdisciplinary aspects of the Mathematics in the domain of the art, design, architecture and education, and the relationship between them.

Dirk Huylebrouck has a PhD in Linear Algebra from the University of Gent, Belgium. His interests first went to generalized inverses of matrices over rings, but shifted to irrationality proofs for pi and zeta (Lester Ford award for best paper in 'The American Mathematical Monthly' in 2002). It recently led to new generalizations of Wallis' product formulas. Since 1997 he is the editor of the column 'The Mathematical Tourist' in 'The Mathematical Intelligencer'. Perhaps he is better known for the discovery of errors in the work of Leonardo da Vinci, in the Belgian Atomium landmark, in the Forbidden Fruit interpretation of the Van Eyck's Mystic Lamb, or, most recently, in Norbert Francis Attard's Fibonacci artwork. 